\title{
Effects of spike-triggered negative feedback on receptive-field properties
}

\author{
Eugenio Urdapilleta ${ }^{1, *}$ and Inés Samengo ${ }^{2}$ \\ ${ }^{1}$ División de Física Estadística e Interdisciplinaria, \\ Centro Atómico Bariloche, Avenida E. Bustillo Km 9.500, \\ S. C. de Bariloche (8400), Río Negro, Argentina \\ Present address: Cognitive Neuroscience, \\ SISSA, via Bonomea 265, 34136 Trieste, Italy \\ ${ }^{2}$ División de Fúsica Estadística e Interdisciplinaria 6 Instituto Balseiro, \\ Centro Atómico Bariloche, Avenida E. Bustillo Km 9.500, \\ S. C. de Bariloche (8400), Río Negro, Argentina
}

(Dated: Received: date / Accepted: date) 


\begin{abstract}
Sensory neurons are often described in terms of a receptive field, that is, a linear kernel through which stimuli are filtered before they are further processed. If information transmission is assumed to proceed in a feedforward cascade, the receptive field may be interpreted as the external stimulus' profile maximizing neuronal output. The nervous system, however, contains many feedback loops, and sensory neurons filter more currents than the ones representing the transduced external stimulus. Some of the additional currents are generated by the output activity of the neuron itself, and therefore constitute feedback signals. By means of a time-frequency analysis of the input/output transformation, here we show how feedback modifies the receptive field. The model is applicable to various types of feedback processes, from spike-triggered intrinsic conductances to inhibitory synaptic inputs from nearby neurons. We distinguish between the intrinsic receptive field (filtering all input currents) and the effective receptive field (filtering only external stimuli). Whereas the intrinsic receptive field summarizes the biophysical properties of the neuron associated to subthreshold integration and spike generation, only the effective receptive field can be interpreted as the external stimulus' profile maximizing neuronal output. We demonstrate that spike-triggered feedback shifts low-pass filtering towards band-pass processing, transforming integrator neurons into resonators. For strong feedback, a sharp resonance in the spectral neuronal selectivity may appear. Our results provide a unified framework to interpret a collection of previous experimental studies where specific feedback mechanisms were shown to modify the filtering properties of neurons.
\end{abstract}

* eugenio.urdapilleta@cab.cnea.gov.ar 


\section{INTRODUCTION}

Sensory areas are exposed to large variations in the physical magnitudes they encode Dong and Atick 1995, Schwartz and Simoncelli 2001, Geisler 2008, Theunissen et al. 2000]. The dynamic range of input signals can often span several orders of magnitude during the course of a single behaviorally relevant time interval. For example, in the visual system, mean luminosity changes drastically when the gaze is displaced from a spot that is directly illuminated by the sun, to a shadowy corner. Neural systems have therefore developed adaptive mechanisms, modifying the neural code according to the sensory context Ulanovsky et al. 2003, Mante et al. 2005].

Several types of adaptive mechanisms exist, as for example, synaptic plasticity Atwood and Karunanithi 2002, Xu-Friedman and Regehr 2004, Siöström et al. 2008, Feldman 2009], feedback through recurrent connectivity Douglas et al. 1995, Shu et al. 2003, Eytan et al. 2003, Buonomano and Maass 2009], feedback through adaptation currents Wang 1998, Sanchez-Vives et al. 2000, Prescott and Seinowski 2008, Peron and Gabbiani 2009], and intrinsic non-linear effects [Borst et al. 2005]. Different mechanisms operate on different timescales; whereas non-linear mechanisms emerge in a matter of milliseconds, synaptic plasticity usually develops in one or a few seconds. Changes in the intermediate range (hundreds of milliseconds) are mostly due to adaptation currents and recurrent connectivity. At these time scales, adaptivity arises from the dynamics associated to certain processes at cellular and network levels, without the profound reorganization entailed by learning and plasticity.

Adaptive phenomena mediated by intrinsic currents and feedback network connectivity are based on history-dependent spike-evoked activity. They exert their feedback influence mainly by reducing neuronal gain [Felsen et al. 2002, Benda and Herz 2003, Ayaz and Chance 2009, Benda et al. 2010], and thereby, by modifying the input/output relation of the cell, that is, the relation between spiking rate and mean stimulus strength. However, adaptation phenomena go far beyond a mere reduction in firing rates, often involving a dramatic reshaping of the selectivity to time-dependent inputs, and of the statistics of neuronal output. For example, in the olfactory bulb, feedback has been shown to amplify input fluctuations of a specific frequency, and thereby to induce strong oscillations in the output activity [Freeman 1972a, Freeman 1972b, Freeman 1972b], giving rise to 
complex (often chaotic) dynamic behavior [Freeman 1987, David and Friston 2003]. Similar conclusions have been reached in theoretical explorations of interacting neural populations |Wilson and Cowan 1972, Coombes and Laing 2009, Buice et al. 2010, Bressloff 2012, Amit and Brunel 1997, Ledoux and Brunel 2011]. Therefore, although adaptation is usually claimed to have evolved in order to increase the dynamic range of sensory encoding, its effect on the temporal properties of the neural code should not be overlooked: Feedback alters the basic properties of neuronal selectivity (as explored below), and also the temporal evolution of the output, the amount of temporal correlations, the precision and the reliability of neural responses [Ladenbauer et al. 2014, Urdapilleta 2011, Butts et al. 2011].

In the context of spike-evoked feedback, adaptation processes have been shown to modify the selectivity to transient stimulus temporal profiles, enhancing the representation of high-frequency components Benda and Herz 2003, Gigante et al. 2007, Benda and Hennig 2008, Benda et al. 2010]. Such changes become evident when computing the receptive field, or the relevant stimulus directions, by means of reverse correlation techniques Davan and Abbott 2001, Chichilnisky 2001, Samengo and Gollisch 2013]. Several previous studies have demonstrated that adaptation in mean firing rates may or may not be accompanied by changes in receptive fields Enroth-Cugell and Shaplev 1973, Victor 1987, Butts et al. 2011, Baccus and Meister 2002, Sharpee et al. 2011, Garvert and Gollisch 2013]. To our knowledge, there is yet no theoretical framework that allows us to understand why and when receptive fields are expected to be modulated by feedback. Such theoretical framework should be general enough to be applicable to cases where adaptation is mediated by feedback at the network level, or at the level of voltage-dependent ionic conductances and refractoriness, as for example, in the Hodgkin-Huxley model [Samengo et al. 2013] or in a LGN model neuron [Gaudry and Reinagel 2007]. In this work we describe the adaptive changes observed in the stationary and transient encoding properties of linear Poisson models driven by a combination of external stimuli and spike-triggered negative feedback. We theoretically analyze how adaptation modifies the shape of the receptive field, providing a complete spectral and temporal description in the limit where the feedback signal is fully determined by the spiking probability of the cell under study (perfect feedback). Spike-evoked negative feedback is shown to induce divisive gain control, to reshape the receptive field, and to enhance resonant properties. In order to extend these results to the case where feedback 
is a noisy function of the spiking probability, we incorporate stochastic elements into the theory. Finally, we also discuss an extension to non-linear Poisson models.

\section{RESULTS}

\section{A. Theoretical description of perfect feedback}

The main goal of this work is to study how the receptive field of a neuron varies, when feedback processes are incorporated. In addition to the temporal dimension, receptive fields may be defined as a function of a variety of additional dimensions (spatial, frequency, chromatic, chemical) depending on the modality of the sensory system under study (vision, audition, taste, etc.). Since adaptation processes unfold along the temporal domain, for the moment, we restrict the analysis to the temporal profile of receptive fields, and defer to the last section of result the extension to higher-dimensional problems.

Strictly speaking, the concept of receptive field is well defined for linear-nonlinear Poisson models. In order to develop the theoretical framework, we initially restrict to purely linear Poisson models, and later on discuss the extension to the nonlinear case. In the linear case, the probability of generating a spike in the interval $[t, t+\mathrm{d} t]$ under the influence of stimulus $I(t)$ is $r(t) \mathrm{d} t$, with

$$
r(t)=h_{0}+\int_{-\infty}^{\infty} h(\tau) I(t-\tau) \mathrm{d} \tau .
$$

Here, $h_{0}$ is the spontaneous firing probability, and $h(\tau)$ is the receptive field of the cell. Since only past stimuli can influence the present firing probability, causality imposes that $h(\tau)=0$, for all $\tau<0$. The stimulus $I(t)$ can be interpreted as either the external signal controlled by the experimentalist (light, sound, touch, etc.), or as the input ionic current entering the cell. By stimulating the cell with stochastic input signals, the shape of $h(\tau)$ may be easily obtained through reverse correlation techniques [Chichilnisky 2001, Samengo and Gollisch 2013]. The shape, units and dimensionality of the filter $h(\tau)$ naturally depend on whether the input signal used in the reverse correlation analysis is the macroscopic external stimulus, or the microscopic ionic current. We specifically distinguish between the component of $I(t)$ representing the transformation of the external signal accomplished by upstream neurons, and the component describing all 
negative feedback signals that are triggered by previous activity of the cell under study

$$
I(t)=s_{0}+s_{1}(t)-g x(t)
$$

In Eq. (2),$s_{0}+s_{1}(t)$ is the extrinsic stimulus component stemming from the transduction of sensory signals, and $x(t)$ is the feedback signal whose value depends on the spiking history of our neuron. The coupling constant $g$ has dimensions of transduced stimulus, and represents the strength of the feedback connection. It may be either negative or positive, depending on whether the cell under study fires in response to positive or negative stimulus deflections. The sign must be chosen in such a way as to produce a signal that opposes the natural excitability of the cell, in order to avoid positive-feedback instabilities (see below). We separate the average external stimulus $s_{0}$, so that the time-dependent component $s_{1}(t)$ can be assumed to have zero mean. Defining the baseline firing rate

$$
r_{0}=h_{0}+H s_{0},
$$

and replacing Eq. (2) in Eq. (11), we obtain

$$
r(t)=r_{0}+\int_{-\infty}^{\infty} h(\tau)\left[s_{1}(t-\tau)-g x(t-\tau)\right] \mathrm{d} \tau
$$

where

$$
H=\int_{0}^{\infty} h(\tau) \mathrm{d} \tau=\sqrt{2 \pi} \hat{h}(\omega=0) .
$$

The parameter $H$, hence, is proportional to the zero-frequency component of the Fourier transform of the receptive field, $\hat{h}(\omega=0$ ) (the convention used for the Fourier transform is specified in Appendix A).

We assume that the feedback signal $x(t)$ is boosted by discrete pulses, and has a natural decay time $\tau_{\mathrm{d}}$. For example, if feedback is implemented through the inhibitory action produced by nearby neurons, the pulses represent spikes produced by other cells in the network providing inhibitory feedback to the neuron under study. Hence,

$$
\frac{\mathrm{d} x}{\mathrm{~d} t}=-\frac{x}{\tau_{\mathrm{d}}}+\sum_{\mathrm{i}=1, \forall \mathrm{k}}^{N} \alpha_{\mathrm{i}} \delta\left(t-t_{\mathrm{i}}^{\mathrm{k}}\right),
$$


where $\alpha_{\mathrm{i}}$ weighs the increase of feedback activity due to a spike in the $i$-th presynaptic neuron, occurred at time $t_{\mathrm{i}}^{\mathrm{k}}$ (k collectively represents all spike times). If Eq. (6) is meant to represent a feedback signal, the average activity of the neurons contributing to the sum must be proportional to the output of the neuron under study. Only with such proportionality can we ensure that $x(t)$ is linked to the past activity of the neuron. For the sake of simplicity, we model the link as a simple proportionality. Moreover, in our first attempt to model feedback, we assume that the sum in Eq. (6) is not proportional to the actual output of the cells, but rather, to the probability $r(t)$ to generate a given output. That is,

$$
\sum_{\mathrm{i}=1, \forall \mathrm{k}}^{N} \alpha_{\mathrm{i}} \delta\left(t-t_{\mathrm{i}}^{\mathrm{k}}\right) \approx r(t) .
$$

This approximation is here called a perfect feedback signal, since $x(t)$ is a deterministic function of $r(t)$; more specifically, it is a leakily integrated copy of $r(t)$. Later on we discuss the case where the feedback signal is a stochastic (as opposed to deterministic) function of $r(t)$, and more accurately describes the actual output of the cell under study. The approximation of perfect feedback is valid if there is a large number of neurons contributing to the sum in Eq. (6), and if all of them have similar statistical and dynamical properties, so that they are all governed by the same firing probability. Alternatively, one may assume that the external input drives a pool of independent neurons, which collectively provides a normalizing signal associated to the common processing of the incoming stimuli [Heeger 1992, Carandini et al. 1997, Carandini and Heeger 2012]. Mathematically, these conditions mean to assume that all the $\alpha_{i}$ are equal, to take $N \rightarrow \infty$, and in order to maintain the total input bounded, additionally scale $\alpha_{i}=1 / N$. With these approximations, replacing Eq. (7) in Eq. (마), we obtain

$$
\frac{\mathrm{d} x}{\mathrm{~d} t}=-\frac{x}{\tau_{\mathrm{d}}}+r(t)
$$

Equations (44) and (8) constitute a closed set: If the external stimulus $s_{0}+s_{1}(t)$ and the filter $h(\tau)$ are known, both the feedback term and the firing probability can be calculated.

The aim of this study is to deduce how feedback affects the filtering characteristics of the neuron. To that end, we now assume that both the external stimulus and the firing probability are known (the latter can be recorded from repeated presentations of the same stimulus), and evaluate whether the input/output relation can still be considered a filtering 

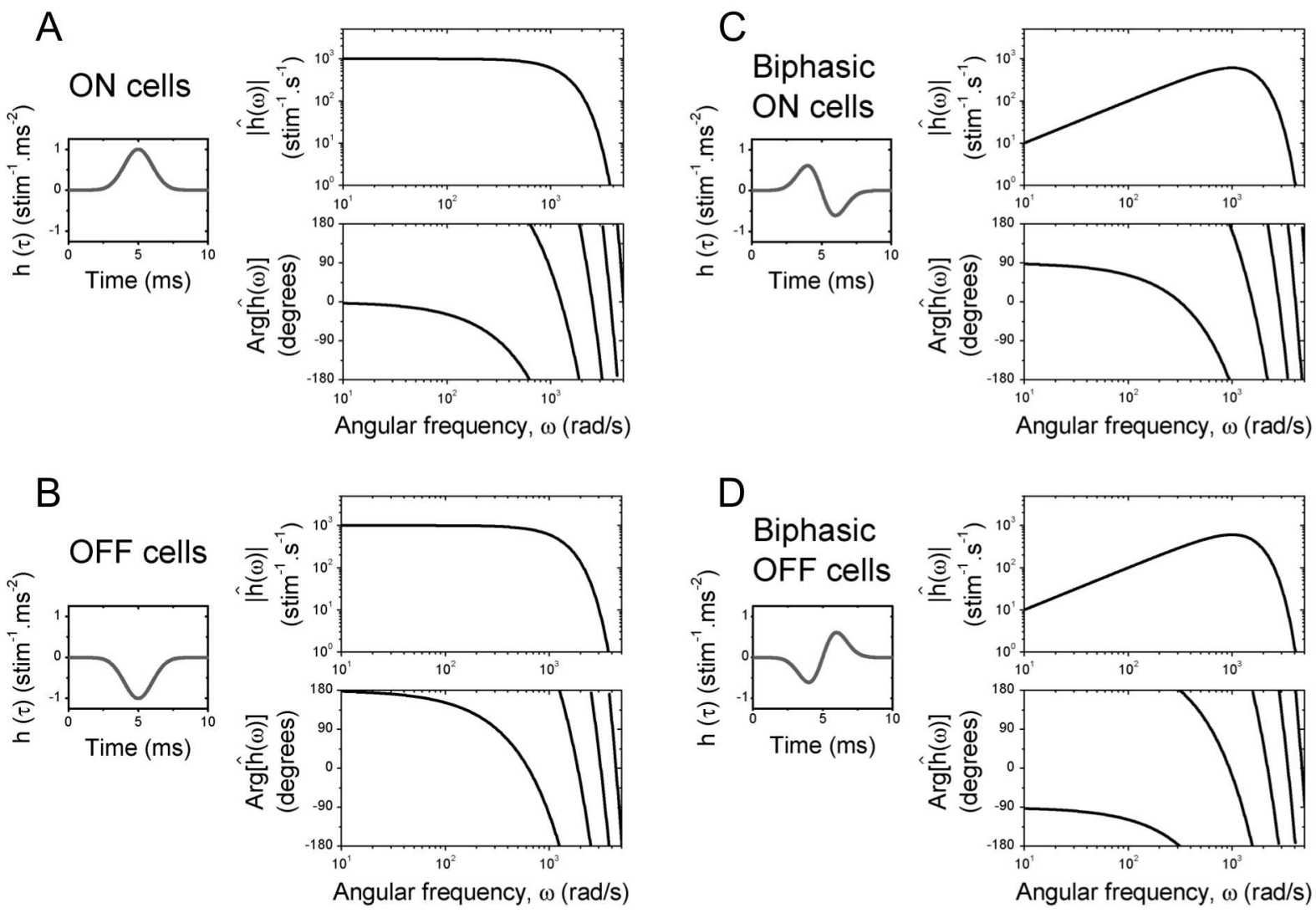

FIG. 1: Spectral characteristics of simplified ON, OFF, and biphasic filters. A: Example of an ON filter displayed as a function of time (left). The modulus and phase of its Fourier transform are displayed as a function of frequency (right). Note the logarithmic and semi-logarithmic scales. $B, C, D$ : Analogous representations of $\mathrm{OFF}$, biphasic-ON and biphasic-OFF filters, respectively. In these examples, ON and OFF filters have exactly the same $|\hat{h}(\omega)|$, and the phases are shifted in $\pi$ (compare $A$ and $B$ ). The same relation is found between biphasic-ON and biphasic-OFF filters (compare $C$ and $D$ ).

process, in spite of feedback. The linear nature of Eqs. (41) and (8) calls for a treatment in Fourier space. Based on the properties of the Fourier transform (see Appendix $\mathrm{A}$ ) and rearranging terms, we obtain

$$
\begin{aligned}
& \hat{r}(\omega)=\frac{\sqrt{2 \pi}\left(1+i \omega \tau_{\mathrm{d}}\right)}{1+i \omega \tau_{\mathrm{d}}+\sqrt{2 \pi} g \tau_{\mathrm{d}} \hat{h}(\omega)} \\
& \times\left[r_{0} \delta(\omega)+\hat{h}(\omega) \hat{s}_{1}(\omega)\right] .
\end{aligned}
$$

In the absence of feedback $(g=0)$, this expression reduces to

$$
\hat{r}(\omega)=\sqrt{2 \pi}\left[r_{0} \delta(\omega)+\hat{h}(\omega) \hat{s}_{1}(\omega)\right] .
$$


Solving Eq. (10) for the filter at non-zero frequencies leads to

$$
\begin{aligned}
\hat{h}(\omega) & =(1 / \sqrt{2 \pi}) \hat{r}(\omega) / \hat{s}_{1}(\omega) \\
& =(1 / \sqrt{2 \pi}) \hat{r}(\omega) \hat{s}_{1}^{*}(\omega) /\left|\hat{s}_{1}(\omega)\right|^{2}
\end{aligned}
$$

so for white-noise stimuli, the filter in the temporal domain $h(\tau)$ is proportional to the spike-triggered average [Gabbiani and Koch 1998].

When feedback is active $(g \neq 0)$, the filter $h(\tau)$ can no longer be calculated with Eq. (11). Comparing Eqs. (9) and (10), we see that for each frequency $\omega$, the firing probability is still a linear function of the applied stimulus. The constant of proportionality, however, is modified due to feedback, and the modification affects differently the continuous component $(\omega=0)$ and the non-zero frequencies $(\omega \neq 0)$. For the continuous case, feedback changes the baseline firing rate $r_{0}$ to an effective value

$$
r_{0}^{\mathrm{fb}}=\frac{h_{0}+H s_{0}}{1+g \tau_{\mathrm{d}} H} \equiv h_{0}^{\mathrm{fb}}+H^{\mathrm{fb}} s_{0},
$$

which implements a divisive gain rescaling.

For non-zero frequencies, feedback modifies the coding properties of the neuron in such a way that the intrinsic filter $h(\tau)$, processing both the external stimulus and the feedback

signal, is equivalent to an effective filter $h^{\mathrm{fb}}(\tau)$ that only filters the external signal. In Fourier space, the relation between the intrinsic and the effective filters is

$$
\hat{h}^{\mathrm{fb}}(\omega)=\frac{1+i \omega \tau_{\mathrm{d}}}{1+i \omega \tau_{\mathrm{d}}+\sqrt{2 \pi} g \tau_{\mathrm{d}} \hat{h}(\omega)} \hat{h}(\omega) .
$$

In the remaining part of the present section, we analyze the effects of Eqs. (12) and (13) both in the frequency and the temporal domains, for several types of filters. We then extend the analysis to imperfect feedback processes, where the sum in Eq. (66) is no longer deterministically proportional to $r(t)$. Finally, we consider non-linear Poisson neurons, and we discuss the validity of the linear approximation.

\section{Poisson neuron models in the absence of feedback}

In order to understand the effect of feedback, we first describe the basic types of processing in a purely feedforward model. Typically, neuronal filtering characteristics are classified 

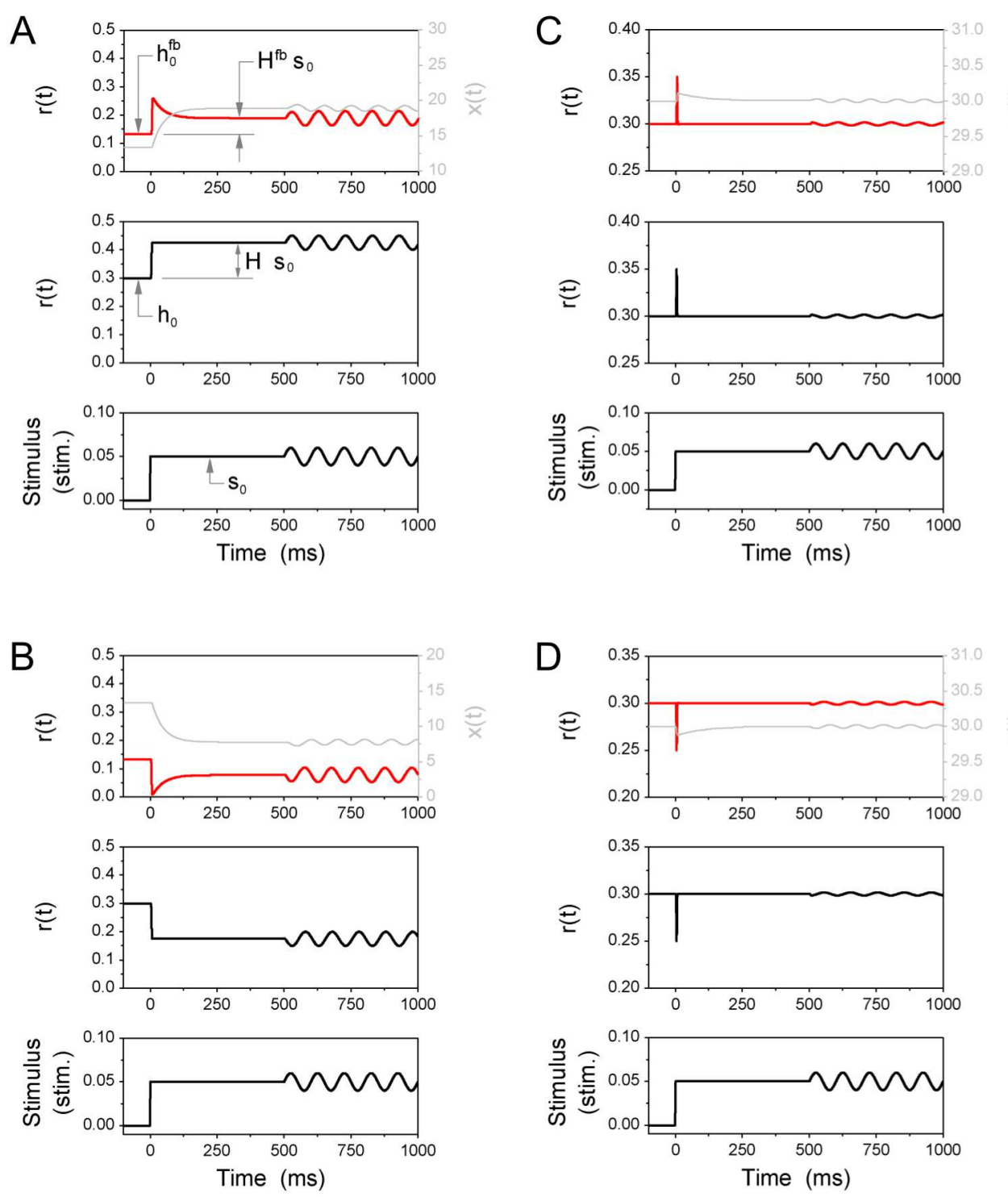

FIG. 2: Temporal evolution of the firing probability of different cells, with and without feedback. $A-D$ : ON, OFF, biphasic ON, and biphasic OFF cell models, respectively (see Fig. 11). Bottom panel: Applied stimulus. At time $t=0 \mathrm{~ms}$, a step of amplitude $s_{0}=0.05$ stimulus units is applied. At time $t=500 \mathrm{~ms}$, the sinusoidal stimulation begins (frequency $10 \mathrm{~Hz}$ and amplitude 0.01 stimulus units). Intermediate panel: Firing probability in the absence of feedback. Top panel: Firing probability $r(t)$ (continuous red line; scale on the left margin) and feedback process $x(t)$ (continuous gray line; scale on the right margin) for the model with negative feedback. Shared parameters: $g=0.005$ stimulus units, $\tau_{\mathrm{d}}=100 \mathrm{~ms}$. Monophasic filters are characterized by $|H|=$ $2.506(\mathrm{stim})^{-1} .(\mathrm{ms})^{-1}$, whereas biphasic filters are symmetric, $H=0$. In all cases, $h_{0}=300 \mathrm{~Hz}$ and the response is measured in $(\mathrm{ms})^{-1}$. For OFF cells (both monophasic and biphasic) the coupling factor $g$ is negative.

according to the shape of $h(\tau)$. For example, in visual areas, the filters of simple cells are classified in a limited number of types [Segev et al. 2006] : ON, OFF, biphasic ON and 
biphasic OFF cells, as shown in Fig. 1. In a previous study [Urdapilleta and Samengo 2009], we demonstrated that when processing slow stimuli, the firing probability of these four different cells is a simple function of the external stimulus. In ON and OFF cells, $r(t)$ is proportional to $s_{1}(t-\delta)$, where the delay $\delta$ is determined by the shape of $h(\tau)$. In biphasic cells, $r(t)$ is proportional to $s_{1}^{\prime}(t-\delta)$, where $s_{1}^{\prime}$ is the temporal derivative of the stimulus. In Appendix B, we offer a novel derivation of these results, based on the Fourier approach developed in this paper.

\section{Poisson neuron models with perfect feedback}

To study the effects of feedback, we separate the analysis in two: First, we focus on the mean response, determined by the spectral content of the response at $\omega=0$. Later, we analyze non-zero frequencies, $\omega \neq 0$.

\section{Effect of feedback on the baseline firing level}

As stated in Eq. (12), the presence of feedback reduces divisively the firing probability. In Fig. 2 we observe how the magnitude of the reduction depends on the type of cell. In the time interval between 0 and $500 \mathrm{~ms}$, the response to a constant stimulus is displayed. Before the onset of the step stimulus $(t<0)$, the presence of feedback $(g \neq 0$, red curve, upper panels) reduces the steady state firing probability, as compared to the value obtained in the absence of feedback ( $g=0$, middle panel), for ON cells (Fig. 2A). The same holds for the steady-state firing probability after the step increase in input current, in the interval between $\sim 250$ and $500 \mathrm{~ms}$. The stationary firing level of OFF cells is also diminished (Fig. 2B) by feedback. In this case, negative feedback is implemented through a negative coupling factor, $g<0$, which could, for example, correspond to excitatory feedback from lateral OFF cells. An OFF cell with a positive $g$ value could cause the firing probability to grow unboundedly, and the Fourier transforms to become ill-defined. For biphasic symmetric cells, the integral of the filter is zero, $H=0$, so feedback does not modify the asymptotic processing of stationary signals (see Figs. $2 C$ and $2 D$, for $t<0$ ).

\section{Effect of feedback on temporal processing}

Combining the effective baseline level $r_{0}^{\mathrm{fb}}$ of Eq. (12) and the effective filter $\hat{h}^{\mathrm{fb}}(\omega)$ defined 
in Eq. (13), we may now re-write Eq. (91) as

$$
\hat{r}(\omega)=\sqrt{2 \pi}\left[r_{0}^{\mathrm{fb}} \delta(\omega)+\hat{h}^{\mathrm{fb}}(\omega) \hat{s}_{1}(\omega)\right],
$$

which is formally equal to Eq. (10). As a consequence, in the time domain,

$$
r(t)=r_{0}^{\mathrm{fb}}+\int_{-\infty}^{\infty} h^{\mathrm{fb}}(\tau) s_{1}(t-\tau) \mathrm{d} \tau .
$$

The intrinsic filter $h(\tau)$ characterizes the biophysical properties of the cell under study. Since $h(\tau)$ filters both the external component of the stimulus $s_{0}+s_{1}(t)$ and the feedback signal $x(t)$, its shape cannot be calculated through reverse correlation analysis performed with solely the external signal; knowledge of the internal signal is also required. The effective filter $h^{\mathrm{fb}}(\tau)$, instead, can be obtained with the sole knowledge of $s_{1}(t)$. Once $\hat{h}^{\mathrm{fb}}(\omega)$ is obtained, the intrinsic filter $\hat{h}(\omega)$ can be recovered with Eq. (13), assuming that feedback properties are known. The temporal profile of the effective filter $h^{\mathrm{fb}}(\tau)$ can be obtained simply by inverse Fourier transform.

The Fourier spectrum of the intrinsic filters of both monophasic and biphasic cells decays at large frequencies (see Fig. 1). Hence, in the high frequency range, the denominator in Eq. (13) is approximately equal to $1+i \omega \tau_{\mathrm{d}}$, and consequently, $\lim _{\omega \rightarrow \infty}\left|\hat{h}^{\mathrm{fb}}(\omega)\right| \approx|\hat{h}(\omega)|$. Biphasic filters also have a reduced spectral content in the low frequency range (see Figs. 1 C and 1D). Therefore, in these cells, feedback exerts a limited effect in the whole frequency range. Since the filtering properties of these cells are hardly modified by feedback, hereafter we focus on monophasic cells (ON and OFF).

In Fig. 3, we discuss the differences in the spectral domain between $\hat{h}^{\mathrm{fb}}(\omega)$ and $\hat{h}(\omega)$. For any OFF filter there is another ON filter that is exactly equal in shape, but with inverted sign. To consider negative feedback, the coupling constant $g$ acting on an OFF cell also has to be inverted in sign, otherwise, we fall on the case of positive feedback, which is unstable in the linear case. Hence, due to inversion symmetry, ON and OFF cells present exactly the same gain spectral behavior. Additionally, filter phases of ON and OFF cells display the same spectral characteristics but shifted in $\pi$.

Overall, for monophasic cells, we observe a moderate spectral reshaping of filters due to the presence of feedback. The low-pass filtering characteristic of $\hat{h}(\omega)$ are converted to band-pass filtering properties in $\hat{h}^{\mathrm{fb}}(\omega)$ (see black and red continuous lines, respectively, in 

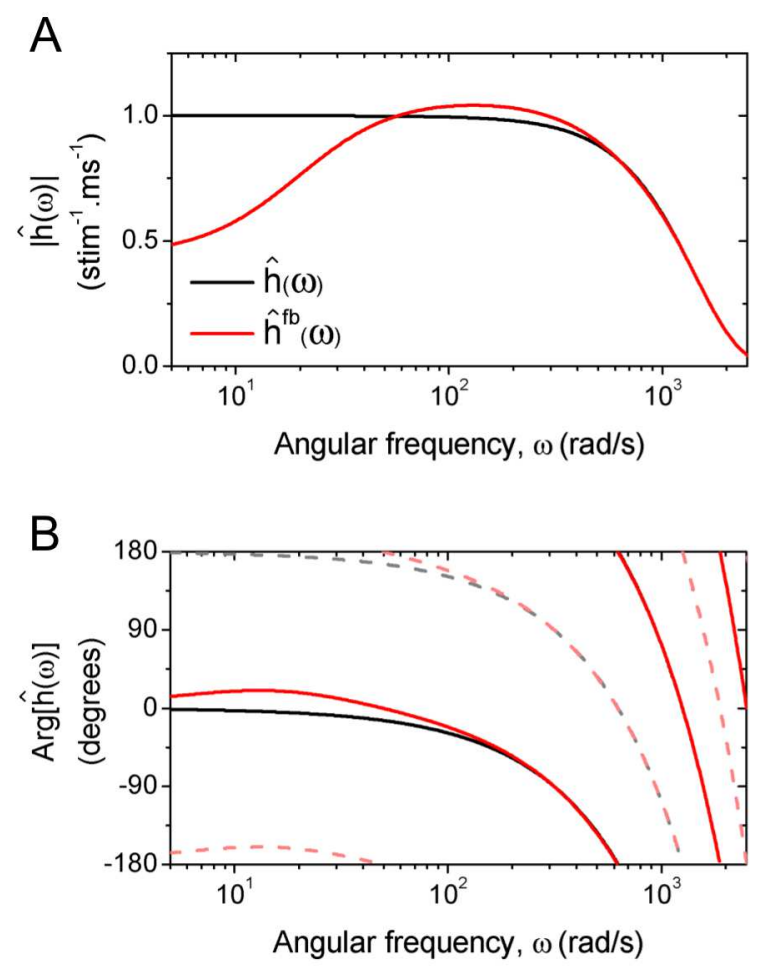

FIG. 3: Spectral composition of filters of monophasic cells, with and without feedback $\left(\hat{h}^{\mathrm{fb}}\right.$ in red and $\hat{h}$ in black lines, respectively). A: The modulus $|\hat{h}(\omega)|$ is exactly the same for ON and OFF cells. Feedback reduces the spectral content at low frequencies and induces a relative increase in the intermediate range. B: Phase of $\hat{h}^{\mathrm{fb}}(\omega)$ and $\hat{h}(\omega)$, for an ON cell (full lines) and an OFF cell (dashed lines). Feedback advances the phases at intermediate frequencies. Parameters as in Fig. 2 ,

Fig. 3A). Feedback reduces the contribution of low frequency stimuli and slightly enhances the influence of stimuli with intermediate frequencies. Since the system is linear, the application of a sinusoidal stimulus (see Fig. 2, for $t>500 \mathrm{~ms}$ ) evokes a sinusoidal response probability; the ratio between relative amplitudes of the response and the stimulus (multiplied by a factor $1 / \sqrt{2 \pi}$ ) defines the gain or the modulus of the filter. The introduction of negative feedback produces a minor effect on the phase. Importantly, however, a phase advance is observed at intermediate frequencies, implying that $r$ overtakes $s$ in the asymptotic regime (see Fig. 2 for $t \gg 500 \mathrm{~ms}$ ).

Feedback is defined by two parameters: the time constant $\tau_{\mathrm{d}}$ that determines the temporal development of $x(t)$, and its relative contribution to the input signal, given by $g$. In Figs. 4 $A$ and $4 C$ we study the effect of varying the coupling strength $g$ on a monophasic ON filter. When $g$ is small, feedback barely influences neural processing. As $g$ increases, low frequency content decreases in order to satisfy $\hat{h}^{\mathrm{fb}}(\omega) \rightarrow H^{\mathrm{fb}} / \sqrt{2 \pi}=\hat{h}(0) /\left(1+g \tau_{\mathrm{d}} H\right)$ and band-pass 
A

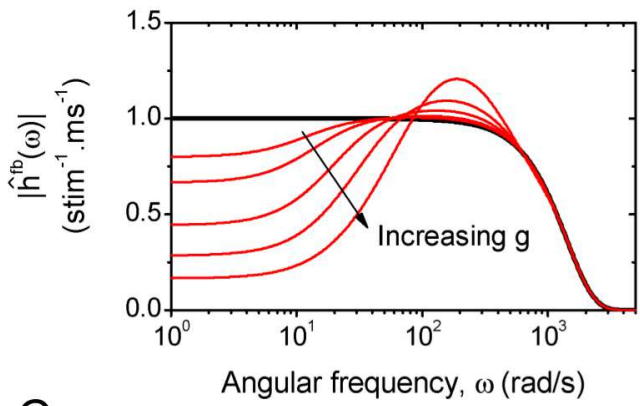

C

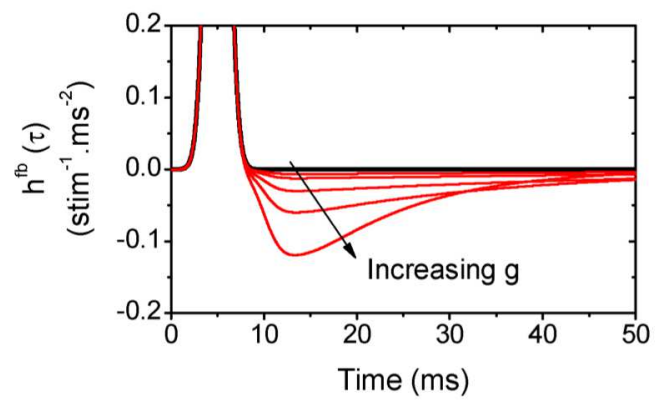

B

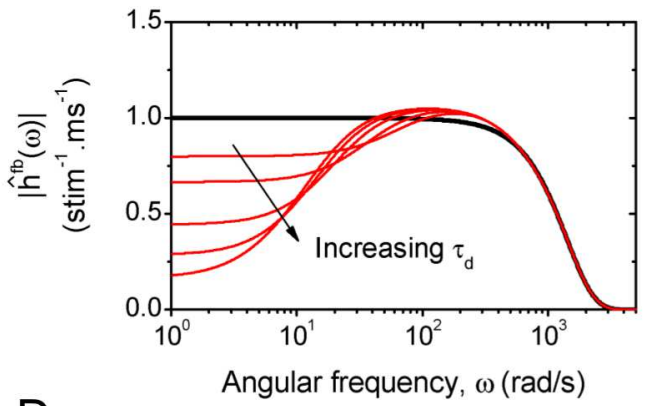

$\mathrm{D}$

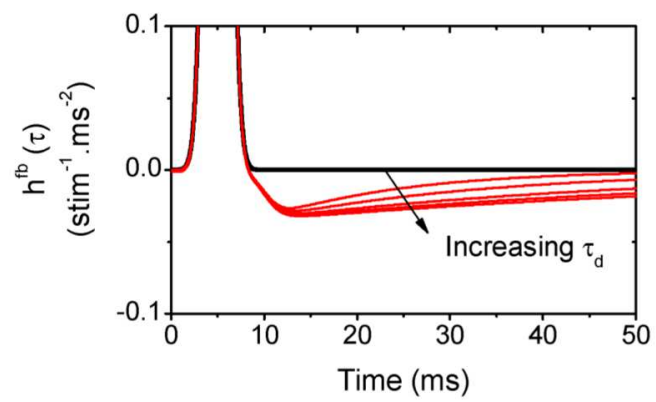

FIG. 4: Influence of parameters defining feedback on spectral and temporal processing. Black line: intrinsic filter $h$. A: Modulus of the feedback filter $\hat{h}^{\mathrm{fb}}(\omega)$, for different values of the coupling strength $g$ (red lines), with $g=1,2,5,10$ and $20 \times 10^{-3}$ stimulus units. For all cases, $\tau_{\mathrm{d}}=$ $100 \mathrm{~ms}$. B: Modulus of the feedback filter $\hat{h}^{\mathrm{fb}}(\omega)$, for different time constants $\tau_{\mathrm{d}}$ (red lines), with $\tau_{\mathrm{d}}=20,40,100,200$ and $400 \mathrm{~ms}$. For all cases, $g=5 \times 10^{-3}$ stimulus units. $C$, D: Temporal filter reconstructed from the spectra in $A$ and $B$ and the corresponding phases (not shown). $C$ : As $g$ increases, the region of inverted polarity becomes more prominent. $D$ : As $\tau_{\mathrm{d}}$ increases, the recovery from the inverted polarity becomes slower. In all cases, $H=2.506(\mathrm{stim})^{-1} \cdot(\mathrm{ms})^{-1}$.

behavior is emphasized. To see how these characteristics appear in the temporal domain, we calculate the filter $h^{\mathrm{fb}}(\tau)$ by applying the inverse Fourier transform on $\hat{h}^{\mathrm{fb}}(\omega)$. The effective filter $h^{\mathrm{fb}}(\tau)$ is no longer purely monophasic, as the original filter $h(\tau)$, since it contains a late phase of inverted polarity (see Fig. 46 ). The absolute value of the integral of the filter is also reduced, when compared to the original $H$. Therefore, the feedback filter is more sensitive to the fluctuations of the stimulus than the original filter [Urdapilleta and Samengo 2009]. As the coupling strength $g$ grows, stronger feedback generates a more significant region of inverted polarity in $h^{\mathrm{fb}}(\tau)$.

The effect of varying $\tau_{\mathrm{d}}$ is shown in Figs. $4 B$ and $4 D$. For fixed $g$, if $\tau_{\mathrm{d}}$ increases, low frequency components decrease (Fig. 4B). In addition, for small values of $\tau_{\mathrm{d}}$, the gain extends its zero-frequency value, $H^{\mathrm{fb}} / \sqrt{2 \pi}$, into a wider range of positive frequencies. As shown in Fig. $4 B, \tau_{\mathrm{d}}$ has only a minor influence at intermediate frequencies. Finally, in the temporal 
domain, the duration of the region of inverted polarity is governed by $\tau_{\mathrm{d}}$ (see Fig. $4 D$ ).

To summarize, we conclude that whenever reverse correlation reveals a filter with biphasic characteristics, the obtained effective filter $h^{\mathrm{fb}}(\tau)$ may not coincide with the intrinsic filter $h(\tau)$. In particular, at least some of the biphasic filtering characteristics may derive from negative feedback.

\section{Feedback-induced resonances}

A monophasic receptive field behaves as a low-pass filter, with a cutoff frequency determined by the inverse of the duration of the non-zero portion of $h(\tau)$. The effect of feedback is to additionally reduce the response at low frequencies, so the effective filter $\hat{h}^{\mathrm{fb}}(\omega)$ acquires band-pass characteristics. The value of the lower cutoff frequency and the resulting Q-factor depend on the properties of feedback ( $g$ and $\left.\tau_{\mathrm{d}}\right)$. It is therefore conceivable that by appropriately choosing these two parameters, feedback can be shaped as to induce a strong resonance in the system, even up to the point of instability. In Eq. (13), this kind of strong resonance appears as a sharp peak in $\hat{h}^{\mathrm{fb}}(\omega)$, or even a divergence. The denominator of Eq. (13) can indeed vanish for specific combinations of discrete frequencies $\omega_{j}$ and values of the product $\left(g \tau_{\mathrm{d}}\right)_{j}$. In these cases, the presence of negative feedback renders the system unstable, amplifying one particular frequency (or several). Near a resonance, any infinitesimal stimulus component matching the critical frequency is amplified in the response, giving rise to strong oscillations. The oscillations enter repeatedly into the feedback loop producing a divergent response. Of course, no real neuron can truly produce diverging responses, because as oscillations grow in amplitude, Eq. (1) loses validity: The evolution of the system can no longer be described by a linear equation; in particular, the linear scheme must be abandoned before the oscillations in the firing probability $r(t)$ are strong enough as to produce negative values.

The linear analysis is nevertheless useful to point out the dramatic amplifying effect that negative feedback can have, and the conditions that favor resonances. Clearly, if the denominator of Eq. (13) vanishes for one or more frequencies, the resulting effective filter $\hat{h}^{\mathrm{fb}}(\omega)$ cannot be transformed back into the temporal domain. As stated above, such divergences may appear (if at all) at a discrete collection of frequencies $\omega_{j}$, and discrete values of the

product $\left(g \tau_{\mathrm{d}}\right)_{j}$. The absence of divergences, however, does not guarantee that the inverse 

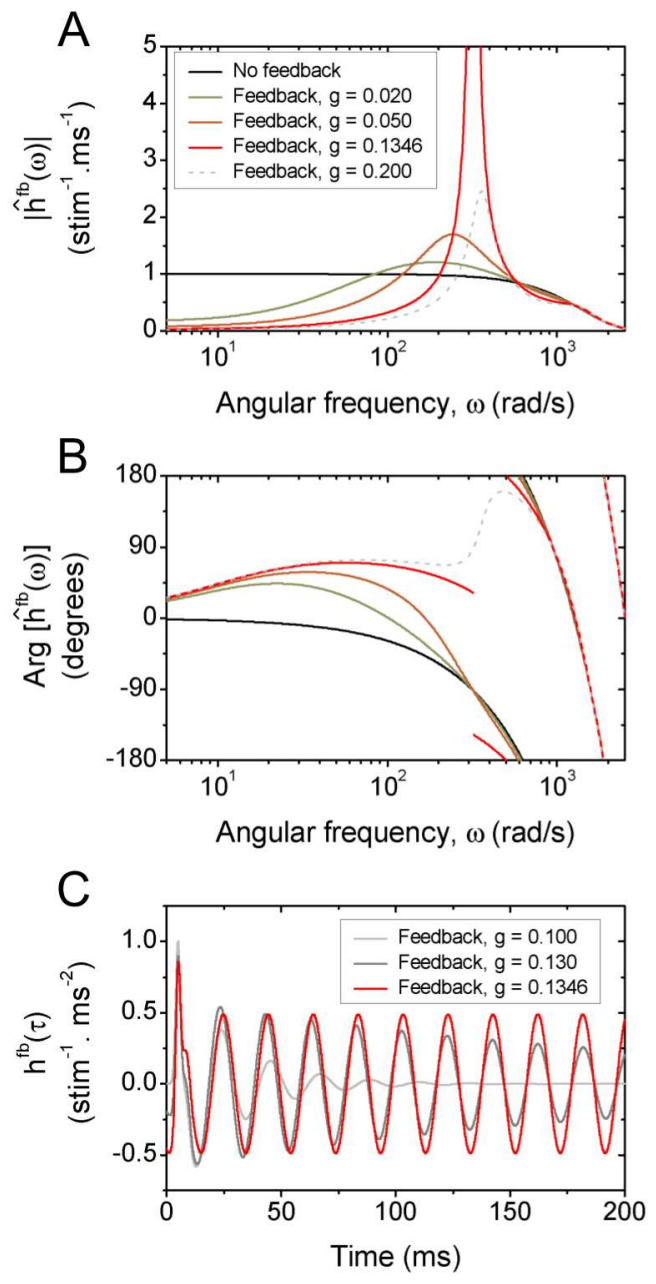

FIG. 5: Feedback-induced resonances for the ON filter of Fig. 1. A: Modulus of the effective filter $\left|\hat{h}^{\mathrm{fb}}(\omega)\right|$ near the resonant instability. For negative feedback with timescale $\tau_{\mathrm{d}}=100 \mathrm{~ms}$, a sharp peak develops at $\omega \approx 320.4 \mathrm{rad} / \mathrm{s}$. In this case, the critical value for the coupling strength is $g_{i} \approx 0.1346$ stimulus units. $B$ : The phase of the effective filter varies rapidly near the resonance. $C$ : In the temporal domain, as the resonance is approached, the effective filter develops strong oscillations at the frequency of the critical $\omega_{i}$. For $g>g_{i}$, the filter $\hat{h}^{\mathrm{fb}}(\omega)$ cannot be transformed back to the time domain, implying that the linear system of Eq. (1) is ill defined.

Fourier transforms $h^{\mathrm{fb}}(\tau)$ and $r(t)$ exist and are bounded. In fact, the condition that gives rise to instabilities is broader and includes the discrete cases where the denominator of Eq. (13) vanishes. The proper mathematical framework to analyze the onset of instabilities is provided by control theory [Franklin et al. 1994], and can be addressed in terms of the behavior of the Laplace transform of the effective filter $\tilde{h}^{\mathrm{fb}}(s)$ with complex argument $s=\sigma+i \omega$. As derived in Appendix $\mathrm{A}$, an instability appears when at least one pole $s_{j}=\sigma_{j}+i \omega_{j}$ has positive $\sigma_{j}$. The pole $s^{*}$ with largest real part is hence critical, since the 


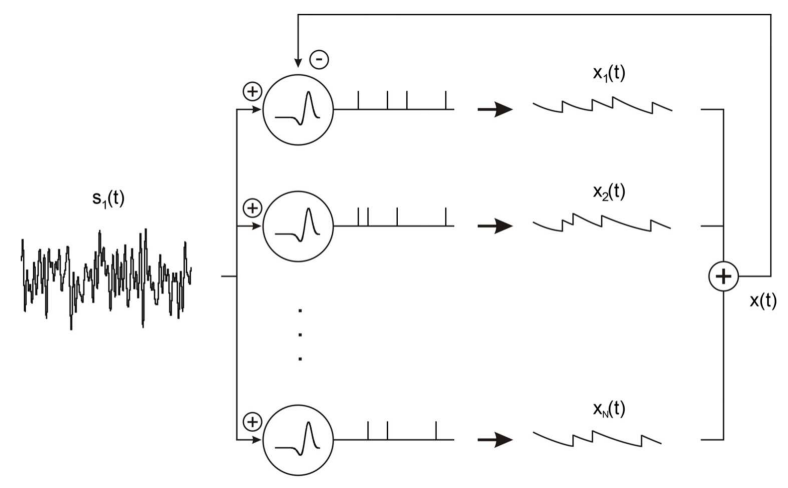

FIG. 6: Inhibitory feedback supported by a finite population of neurons. The signal $s_{1}(t)$ stimulates a population of identical neurons. The output spikes are filtered during synaptic transmission and then return in the form of a negative feedback input current.

magnitude of $\sigma^{*}$ determines the stability of the system. When $\sigma^{*}<0$, the effective filter is qualitatively similar to the examples shown in Figs. 3 and 4 . As the coupling strength $g$ increases, $s^{*}$ gradually shifts to the right and gets closer to the imaginary axis; consequently, the effective filter begins to show a prominent peak at the frequency $\omega^{*}$ of the critical pole, as shown in Fig. [5. This resonance is caused by feedback.

At the resonance, $\omega \approx \omega^{*}$, the phase of $\hat{h}^{\mathrm{fb}}(\omega)$ varies rapidly (Fig. $5 B$ ). In the temporal domain, the effective filter exhibits strong oscillations (Fig. [5C), which occasionally grow as far as to make the system unstable. A mathematical analysis of the conditions giving rise to instability (see Appendix A) reveals that unstable behavior is only observed if $g$ is above a critical threshold, the value of which is determined by the shape of the intrinsic filter $\hat{h}(\omega)$. For large $g$, however, the baseline firing level $r_{0}^{\mathrm{fb}}$ drops significantly (see Eq. (12)). Therefore, a transition to instability may only be expected in systems with strong feedback and, simultaneously, with large intrinsic spontaneous activity $r_{0}$ or decreased stimulus fluctuations $s_{1}(t)$, so as to ensure that the firing rate $r(t)$ remains positive. Once these conditions are met, the frequency $\omega^{*}$ of the unstable oscillations is determined by the time constant $\tau_{\mathrm{d}}$ and the shape of the intrinsic filter $h(\tau)$. In the limit of large $\tau_{\mathrm{d}}$ (as in the example of Fig. 5 ), the feedback time constant becomes irrelevant, and the location of the critical frequency depends only on $h(\tau)$. 


\section{B. Poisson neuron models with imperfect feedback}

So far, the analysis was based on Eqs. (41) and (8), where the temporal derivative of the feedback signal $x(t)$ was proportional to the firing probability $r(t)$. This relation is only valid in the limit of a homogeneous population of infinitely many identical neurons, uniformly coupled and driven with identical stimuli $s(t)$. These conditions are hardly realistic, since the amount of feedback must be determined by the actual number of generated spikes, and not by the spiking probability $r(t)$. There is an important difference between these two options. The spiking probability $r(t)$ is a deterministic function of the input current $I(t)$ (Eq. (1)), whereas actual spikes are stochastic point processes governed by $r(t)$. Therefore, in an attempt to provide a more realistic description of feedback, we now assume that $x(t)$ is given by Eq. (6) . Although this new model can be studied analytically in specific parameter regimes (see below), we initially resort to a numerical approach to determine the point up to which the results of the previous section can be extended to more realistic conditions.

In the present description, illustrated in Fig. 6, we assume that neurons are limited in number, all have identical intrinsic filtering properties $h(\tau)$, and process a common temporal stimulus, $s_{1}(t)$. Furthermore, the feedback signal $x(t)$ is assumed to be the same for all cells, so the firing probability $r(t)$ given by Eq. (4) holds for any cell in the population. However, since spike generation in Poisson processes is stochastic, the precise temporal location of spikes differs from neuron to neuron and, therefore, the sum in Eq. (66) is a random variable, which only recovers its deterministic limit (Eq. (8) ) when the number of neurons tends to infinity $(N \rightarrow \infty)$. Since the derivative of $x(t)$ is no longer strictly proportional to $r(t)$, feedback is now called imperfect.

In Fig. 77A we show the evolution of the firing probability $r(t)$ (upper panel), along with the feedback signal $x(t)$ (middle panel) and the driving stimulus (bottom panel) for the finite population model. These three signals are common to all neurons. The feedback signal is the sum of $N$ filtered spike trains, a few of which are displayed in Fig. $7 B$. The discontinuities in the traces (inset) are produced by individual spikes. These irregular traces barely resemble the deterministic counterpart $x_{\text {det }}(t) / N$ obtained in the limit $N \rightarrow \infty$ (black curve). Their sum, however, smoothes fluctuations out, and follows $x_{\operatorname{det}}(t)$ closely (middle panel of Fig. [7A).

To quantify the stochastic behavior of this system, we drive the cells with a sinusoidal 

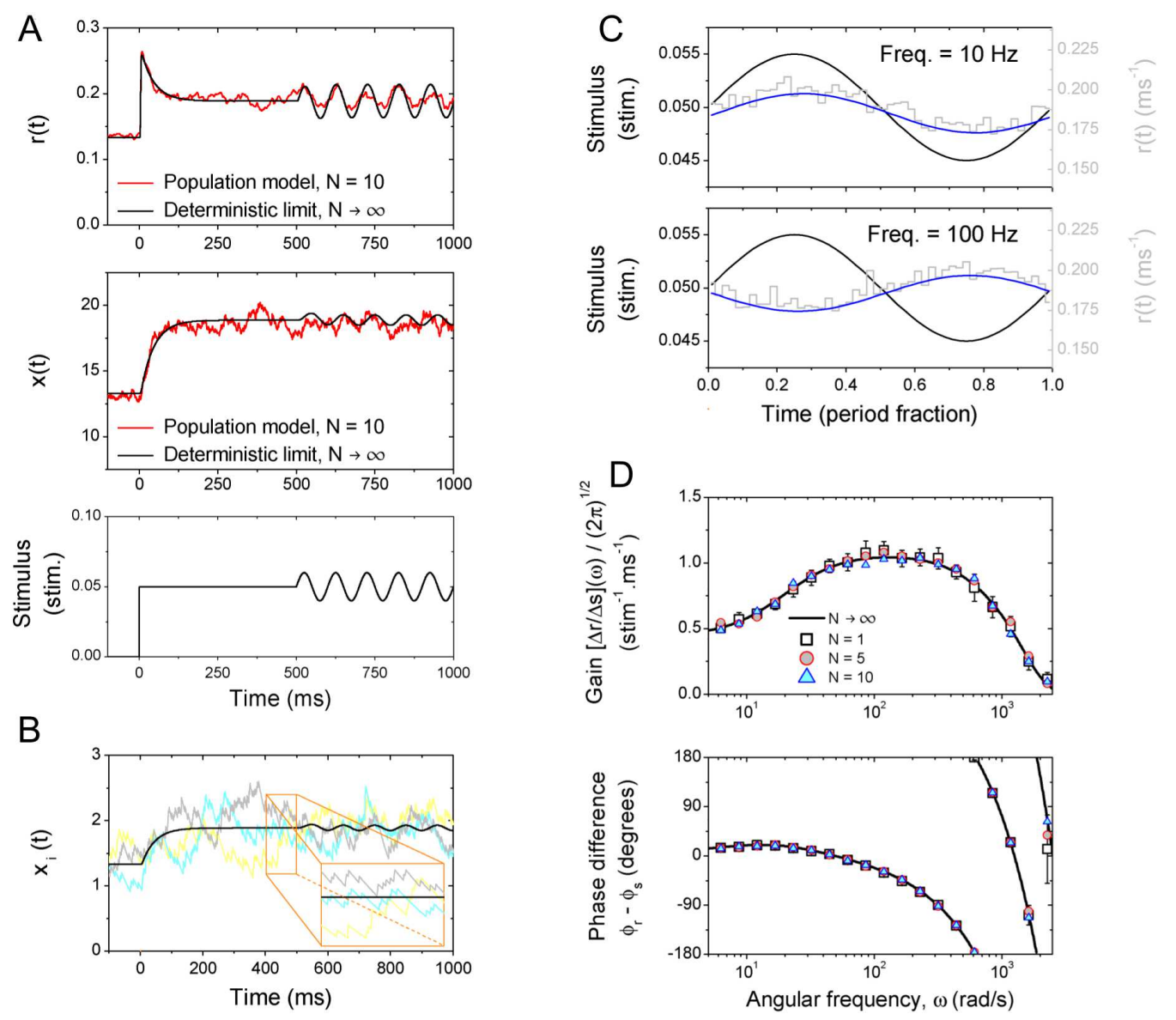

FIG. 7: Evolution of dynamic variables in the presence of imperfect feedback. A: Time-dependent firing probability $r(t)$ and feedback process $x(t)$ for the model with imperfect feedback driven by the stimulus $s_{0}+s_{1}(t)$. Black lines: prediction for $N \rightarrow \infty$. Irregular red lines: single-neuron signals, obtained from a population of $N=10$ neurons. $B$ : Single spike trains filtered with a simple synaptic dynamics, $\mathrm{d} x_{\mathrm{i}} / \mathrm{d} t=-x_{\mathrm{i}} / \tau_{\mathrm{d}}+(1 / N) \delta\left(t-t_{\mathrm{i}}^{k}\right)$. Individual signals in colored irregular lines. Irregularity arises from the discontinuities evoked by incoming spikes, amplified in the inset. The feedback signal $x(t)$ shown in $A$ (middle panel) is $\sum_{i=1}^{N} x_{\mathrm{i}}(t)$ (notice the scale difference with $x_{\mathrm{i}}(t)$ in $B$ ). Black line: proportional contribution of a single $x_{\mathrm{i}}(t)$ in the limit $N \rightarrow \infty$. C: Extracting the relation between stimulus and response in noisy conditions. For a linear system, a small sinusoidal stimulus (black line; scale of the left) produces a sinusoidal firing probability of identical frequency (gray line, scale on the right). A long run of the output spike train is split in $T$-windows, where $T$ is the period of the sinusoidal input. All spikes are then wrapped in a single window, and the histogram $r(t)$ is constructed. Top/bottom panels: Stimulation at $10 \mathrm{~Hz} / 100 \mathrm{~Hz}$. D: From the fit of the histograms in $C$, we calculate the gain $\frac{1}{\sqrt{2 \pi}} \frac{\Delta r}{\Delta s}$, and the phase, $\phi_{r}-\phi_{s}$, of the transfer function $(\Delta r$ is the amplitude of the sinusoidal function that best fits the response). Different symbols represent different population sizes. Spikes are collected in asymptotic conditions (that is, after the initial transient) during $500 \mathrm{~s}$. For each data point, 10 repetitions are simulated. Error bars indicate the standard deviation of the gain and phase, as calculated from the best fit parameters obtained for each of these repetitions. Parameters: $H=2.506(\mathrm{stim})^{-1} \cdot(\mathrm{ms})^{-1}$, $\tau_{\mathrm{d}}=100 \mathrm{~ms}, g=0.005$ stimulus units, $r_{0}=0.3 \mathrm{~ms}^{-1}, s_{0}=0.05$ and $\Delta s=0.005$ stimulus units. 
stimulus. As observed in Fig. $7 \mathrm{~A}$, for times $t>500 \mathrm{~ms}$, the firing probability has a marked periodic component at the frequency of the input signal. Therefore, the input/output properties can be characterized by studying a single stimulus cycle. This reduction is implemented by taking the spikes fired in different time windows (one window per stimulus period $T$ ) and wrapping them together into a single window, taking care of preserving the original firing phase with respect to the stimulus. That is, each spike is displaced an integer number of periods, and located within a window whose duration is equal to a single period of stimulation. Once a long spike train realization is so wrapped, the corresponding histogram in the $T$-window is built, as shown in Fig. $7 C$. In this figure, we can clearly observe the periodic modulation of the response (stairs-like gray lines), which expectedly can be fitted by a sinusoidal function (continuous blue lines). The amplitude and phase (relative to the stimulus) of the adjusted response is used to construct the spectral characteristics of the stochastic model.

In Fig. $7 \mathrm{C}$ we show the spectral characteristics of the population-based feedback model. Both the gain and the phase shift are practically invariant with the number of neurons in the population, and a good agreement with the case $N \rightarrow \infty$ (black line) is observed. The dispersion at each point (error bars) arises from the variability in the sinusoidal fit, which depends on the irregularities of the histogram (controlled by the total recording time and the mean number of spikes produced during a cycle). The irregularities, in turn, arise from the Poissonian character of the spike generation process. When $N=1$, the filtered activity of the neuron itself is used as the feedback signal defined in Eq. (6), and the sum in $i$ just involves a single element, $i=1$. This situation is adequate to model self-inhibition due to spiketriggered adaptation currents [Benda and Herz 2003, Benda et al. 2010, Urdapilleta 2011]. As $N$ grows, the description gradually shifts to represent network-mediated feedback processes.

The wrapping procedure used to construct Fig. 7 allows us to determine the linear response function as the ratio between the amplitude of the firing probability (response) and the stimulus driving the system (input), both measured at the same frequency. One important result is that the linear response function is independent of the population size $N$ (see Fig. 7D). However, the irregularities observed in the temporal response do indeed depend on $N$. To exemplify this behavior, in Figs. $8 A$ and $8 B$ we show the time-dependent firing probability $r(t)$ and the feedback process $x(t)$, for the cases $N=10, N=5$ and $N=1$. 
Irregularities become more prominent as the population size decreases. When the number of neurons is finite, the firing probability contains a certain amount of power at frequencies different from the incident frequency. The power at spurious frequencies does not affect the linear response function, because the fitting procedure is casted specifically at the input frequency. So far we have described the properties of the response at this input frequency. Now we turn our attention to the rest of the spectrum.

As an example, in Figs. $8 \mathrm{D}$ and $8 \mathrm{E}$ we show the power spectral density of $r(t)$ and of $x(t)$, when the system is stimulated with a $10 \mathrm{~Hz}$, small-amplitude sinusoidal signal. Given the linearity of the system, the firing probability and the feedback signal contain a strong component at precisely $10 \mathrm{~Hz}$ (see the peaks in the corresponding spectra). The height of the peak in $r(t)$ is given by the linear response function studied before (see Fig. 7). The remaining spectral power (the background) arises from the inherent randomness of Poisson processes. As the population size increases, fluctuations in the spontaneous regime diminish and, correspondingly, the background spectral density decreases as well.

The remaining part of this section is devoted to obtain an analytical expression of the background spectrum. We first analyze the feedback process corresponding to $N=1$ (adaptation current), and later generalize the result to arbitrary $N$. For $N=1$, the signal $x(t)$ evolves according to

$$
\frac{\mathrm{d} x}{\mathrm{~d} t}=-\frac{x}{\tau_{\mathrm{d}}}+\xi(t)
$$

where $\xi(t)$ represents the spike train produced by a Poisson process (a realization), with a time-dependent rate given by Eq. (4). This rate is coupled to Eq. (16) (and thereby, to the noise source) through the feedback term. By approximating the time-dependent firing rate by its baseline level $r_{0}^{\mathrm{fb}}$ (Eq. (12)), Eq. (16) becomes a standard linearly filtered Poisson process (filtered shot noise). Under this approximation, it is simple to find the formal solution to Eq. (16) and the resulting exponential autocorrelation function,

$$
C_{x}(\tau)=\frac{1}{2} r_{0}^{\mathrm{fb}} \tau_{\mathrm{d}} \mathrm{e}^{-|\tau| / \tau_{\mathrm{d}}}
$$

The power spectral density (one-sided, per unit time) of the feedback signal $x(t)$ can be obtained from the autocorrelation function through the Wiener-Khinchin theorem Gardiner 1985, Press et al. 2007], 

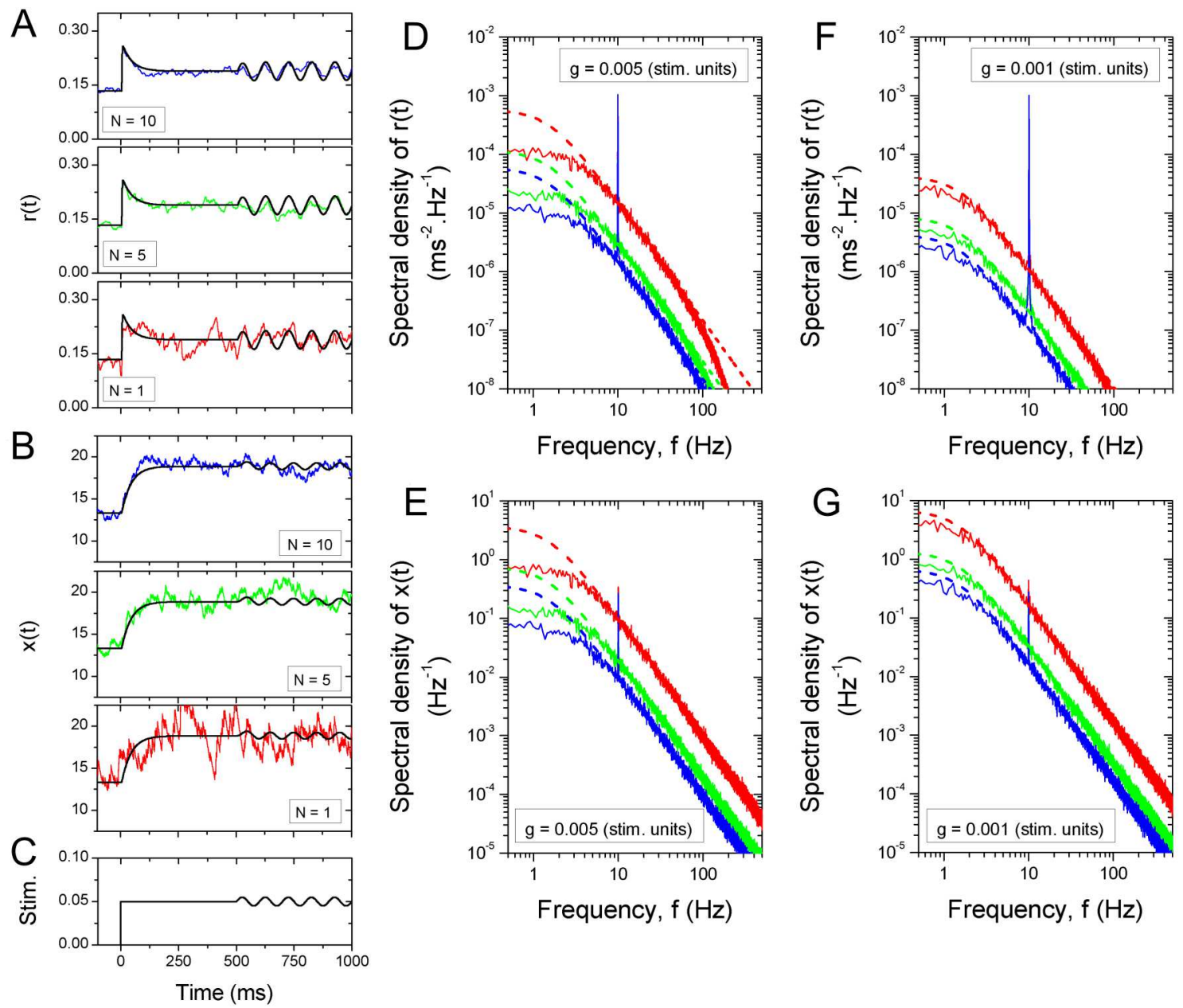

FIG. 8: Characterization of finite size effects. $A, B$ : Evolution of the time-dependent firing rate $r(t)$ (in $\mathrm{ms}^{-1}$ ) and feedback process $x(t)$ (adimensional), for the stimulus in $C$. In $A$ and $B$, different panels correspond to different population sizes: $N=10$ (blue, top panel), $N=5$ (green, middle panel), and $N=1$ (red, bottom panel). Black lines: deterministic limit $N \rightarrow \infty$. All parameters are the same as those used in Fig. 2A. As $N$ decreases, fluctuations are amplified. $D$, E: Power spectral density per unit time of $r(t)(D)$ and $x(t)(E)$, in the asymptotic regime of small-amplitude, periodic stimulation (frequency: $10 \mathrm{~Hz}$, amplitude: 0.005 stimulus units). Colors as in $A$ and $B$. The theoretically derived power spectral density, under the weak coupling assumption, is shown in dashed lines (with corresponding colors) for $S_{r}(f)(D)$, Eq. (25), and $S_{x}(f)(E)$, Eq. (22). F, G: Analogous to panels $D, E$ for a weaker feedback, $g=0.001$ stimulus units.

$$
\begin{aligned}
\frac{S_{x}(\omega)}{T} & =\frac{1}{T}\left[|\hat{x}(-\omega)|^{2}+|\hat{x}(\omega)|^{2}\right] \\
& =\frac{1}{\sqrt{2 \pi}}\left[\hat{C}_{x}(-\omega)+\hat{C}_{x}(\omega)\right] .
\end{aligned}
$$

Since the Fourier transform of Eq. (17) reads 


$$
\hat{C}_{x}(\omega)=\hat{C}_{x}(-\omega)=\frac{r_{0}^{\mathrm{fb}}}{\sqrt{2 \pi}} \frac{1}{\left(1 / \tau_{\mathrm{d}}\right)^{2}+\omega^{2}},
$$

the one-sided power spectral density per unit time is

$$
\frac{S_{x}(\omega)}{T}=\frac{r_{0}^{\mathrm{fb}}}{\pi} \frac{1}{\left(1 / \tau_{\mathrm{d}}\right)^{2}+\omega^{2}}
$$

or, in terms of frequency,

$$
\frac{S_{x}(f)}{T}=2 \pi \frac{S_{x}(\omega)}{T}=\frac{2 r_{0}^{\mathrm{fb}}}{\left(1 / \tau_{\mathrm{d}}\right)^{2}+(2 \pi f)^{2}} .
$$

The previous analysis can be easily extended to the case $N>1$. In this case, the effective rate for $\xi(t)$ in Eq. (16) is now $N r_{0}^{\mathrm{fb}}$. We recall that, in order to maintain the feedback level constant when $N$ increases, the efficacy of each spike in Eq. (6) has to be proportional to $1 / N$. Therefore, now $\mathrm{d} x / \mathrm{d} t=-x / \tau_{\mathrm{d}}+(1 / N) \xi(t)$, and the autocorrelation function is a scaled version of Eq. (17),$C_{x}^{N \text { neurons }}(\tau)=C_{x}^{1 \text { neuron }}(\tau) / N$. Consequently,

$$
\frac{S_{x}(f)}{T}=\frac{1}{N} \frac{2 r_{0}^{\mathrm{fb}}}{\left(1 / \tau_{\mathrm{d}}\right)^{2}+(2 \pi f)^{2}} .
$$

This expression is represented with dashed lines in Fig. $8 E$, for different population sizes. The power spectrum obtained from simulations agrees with the theoretical description, except at low frequencies, where higher order statistical interactions between $x(t)$ and $r(t)$ become noticeable. At medium and high frequencies, the theoretical approach provides a very good description of the simulations.

The firing probability $r(t)$ inherits the correlation structure of $x(t)$,

$$
\begin{gathered}
C_{r}(\tau)=g^{2} \int_{0}^{\tau_{\mathrm{m}}} h\left(\tau^{\prime}\right) \mathrm{d} \tau^{\prime} \int_{0}^{\tau_{\mathrm{m}}} h\left(\tau^{\prime \prime}\right) C_{x}^{(N)}\left(\tau+\tau^{\prime}-\tau^{\prime \prime}\right) \mathrm{d} \tau^{\prime \prime} \\
=\frac{1}{2 N} g^{2} r_{0}^{\mathrm{fb}} \tau_{\mathrm{d}} \int_{0}^{\tau_{\mathrm{m}}} h\left(\tau^{\prime}\right) \mathrm{d} \tau^{\prime} \int_{0}^{\tau_{\mathrm{m}}} h\left(\tau^{\prime \prime}\right) \\
\times \mathrm{e}^{-\left|\tau+\tau^{\prime}-\tau^{\prime \prime}\right| / \tau_{\mathrm{d}}} \mathrm{d} \tau^{\prime \prime}
\end{gathered}
$$

The filter $h(\tau)$ is different from zero inside a finite window [0, $\left.\tau_{\mathrm{m}}\right]$ [Urdapilleta and Samengo 2009]. Therefore, in Eq. (23), we replaced the upper integration limits by $\tau_{\mathrm{m}}$. Whenever $\tau_{\mathrm{m}} \ll \tau_{\mathrm{d}}$, the exponential factor in the integrand of Eq. (23) can be further simplified, so that only time differences $\tau$ comparable or larger than $\tau_{\mathrm{m}}$ matter: 
- $\tau \sim \mathcal{O}\left(\tau_{\mathrm{m}}\right) \Rightarrow \mathrm{e}^{-\left|\tau+\tau^{\prime}-\tau^{\prime \prime}\right| / \tau_{\mathrm{d}}} \approx 1$,

- $\tau \gg \mathcal{O}\left(\tau_{\mathrm{m}}\right) \Rightarrow \mathrm{e}^{-\left|\tau+\tau^{\prime}-\tau^{\prime \prime}\right| / \tau_{\mathrm{d}}} \approx \mathrm{e}^{-|\tau| / \tau_{\mathrm{d}}}$.

In this case, the autocorrelation function can be approximated by

$$
C_{r}(\tau)=\frac{1}{2 N} g^{2} H^{2} r_{0}^{\mathrm{fb}} \tau_{\mathrm{d}} \mathrm{e}^{-|\tau| / \tau_{\mathrm{d}}} .
$$

Finally, based on this equation and the Wiener-Khinchin theorem, the power spectral density for the firing probability $r(t)$ is

$$
S_{r}(f)=\frac{2}{N} \frac{g^{2} H^{2} r_{0}^{\mathrm{fb}}}{\left(1 / \tau_{\mathrm{d}}\right)^{2}+(2 \pi f)^{2}} .
$$

This expression is represented in Fig. $8 D$ for different population sizes, in dashed lines. As in the previous analysis, at low frequencies, the coupling between $x(t)$ and $r(t)$ produces a small discrepancy between the numerical results and the theoretical expression. At high frequencies, a faster decay than the predicted $S_{r}(f) \sim 1 / f^{2}$ is observed, originated by the approximation at $\tau \sim \mathcal{O}\left(\tau_{\mathrm{m}}\right)$ during the assessment of the autocorrelation function $C_{r}(\tau)$.

The derivation in this section is based on the hypothesis that the firing rate $r(t)$ could be approximated by its baseline level $r_{0}^{\mathrm{fb}}$. This assumption is valid if $s_{1}(t)$ is small, and if $x(t)$ and $r(t)$ are weakly coupled. Obviously, as the coupling strength $g$ becomes smaller, the theoretical expressions become more accurate, and are valid in a wider range of frequencies. As shown in Figs. $8 F$ and $8 G$, in this limit, the simulated spectral densities are in excellent agreement with Eqs. (22) and (25)).

\section{Linear-nonlinear Poisson neuron models}

Linear Poisson models are only an approximate description of the processes governing neuronal dynamics. We here improve the approximation by adding a static nonlinearity, as often done in the description of sensory systems [Davan and Abbott 2001, Chichilniskv 2001, Baccus and Meister 2002. Gaudrv and Reinagel 2007, $\quad$ Sharpee et al. 2008, Butts et al. 2011, Garvert and Gollisch 2013]. The new model constitutes the linearnonlinear Poisson approach, where

$$
r(t)=f\left[r_{0}+\int_{-\infty}^{\infty} h(\tau)\left[s_{1}(t-\tau)-g x(t-\tau)\right] \mathrm{d} \tau\right] .
$$


In this section we demonstrate that although the results of the previous section do not strictly hold in the presence of a nonlinearity, it is possible to develop an approximate version of the theory that makes very good predictions in most practical cases.

In Fig. $9 A$, we show three sigmoidal nonlinearities $f$, all with the same functional shape, but with different scaling parameters in the $x$-axis. Here we show how to produce an approximate linearized model, and discuss its range of validity. The first step is to find the operation point, that is, the spontaneous firing rate $r_{0}^{\mathrm{nl}}$, that may differ from the spontaneous rate of the linear case $r_{0}^{\mathrm{fb}}$ (Eq. (12)). In the absence of time-dependent external stimuli, $s_{1}(t)=0$, the linear-nonlinear model reduces to

$$
r_{0}^{\mathrm{nl}}=f\left[r_{0}-g \tau_{\mathrm{d}} H r_{0}^{\mathrm{nl} l}\right] \Rightarrow f^{-1}\left(r_{0}^{\mathrm{nl}}\right)=r_{0}-g \tau_{\mathrm{d}} H r_{0}^{\mathrm{nl}} .
$$

Equation (27) implicitly defines $r_{0}^{\mathrm{nl}}$ as the intersection between the function $f^{-1}\left(r_{0}^{\mathrm{nl}}\right)$ and the straight line $r_{0}-g \tau_{\mathrm{d}} H r_{0}^{\mathrm{nl}}$. In Fig. 9 $B$, we illustrate the procedure. The level $r_{0}$ (arrow on the left margin) sets the offset of the straight line (thick black line). The slope of the line is determined by the intrinsic properties of the neuron $(H)$ and feedback $\left(g \tau_{\mathrm{d}}\right)$. Depending on the steepness of the nonlinearity, one same $r_{0}$ may elicit different spontaneous firing rates $r_{0}^{\mathrm{nl}}$ (colored circles located at the intersections).

If the constant stimulus component $s_{0}$ is modified, a new value $r_{0}$ is established and, after a brief transient evolution, the operation level $r_{0}^{\mathrm{nl}}$ sets to a new value, as predicted by Eq. (27). The $y$-intercept of the straight line shifts, thus displacing the stationary firing probability. In Fig. 9 9 the shift is represented by the two parallel lines (thin gray lines), corresponding to the addition of two constant stimuli of opposite signs. Due to the non-linear nature of the model, the same stimuli displace the response by different amounts (lengths of the error bars). Moreover, positive and negative stimuli produce effects of different magnitude (compare the lengths of the left and the right portions of the error bars).

In Fig. $9 \mathrm{C}$ the temporal evolution of the firing rate (top panel) and the feedback process (middle panel) is shown, for a temporally complex input signal. In the spontaneous regime, $t<0 \mathrm{~ms}$, the firing probabilities obtained in Fig. $9 \mathrm{~B}$ are indicated with colored circles. Following the application of a step stimulus, the firing probability and the feedback process undergo a transient evolution that rapidly settles onto a new stationary value. When the curvature of the nonlinearity is mild, the evolution is similar to the linear case (compare the evolution of the red line in Fig. $9 \mathrm{C}$ with the one of Fig. 2A). As the nonlinearity becomes 

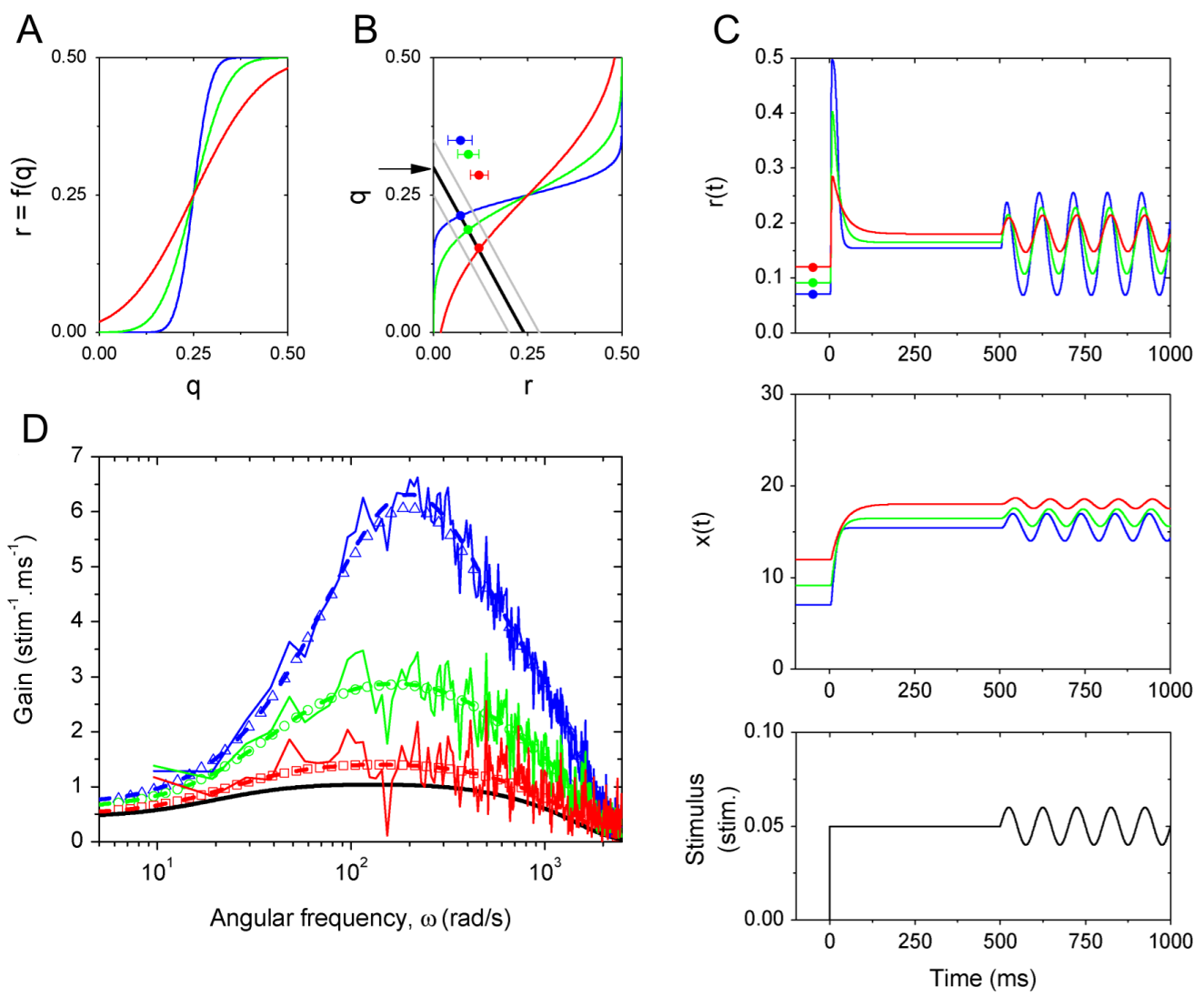

FIG. 9: Effects of a static nonlinearity on the feedback model. A: Sigmoidal nonlinearities modeled as error functions, $(1 / 2) r_{\max }\left\{\operatorname{erf}\left[\left(q-q_{\mathrm{c}}\right) / \Delta\right]+1\right\}$, with $\Delta=0.05, \Delta=0.10$, and $\Delta=0.20$ (blue, green, and red lines, respectively). In all cases, $q_{\mathrm{c}}=0.25$ and $r_{\max }=0.50$. Axis units in $\mathrm{ms}^{-1}$. B: The spontaneous firing rate is defined by the intersection between the line $\left(r_{0}-g \tau_{\mathrm{d}} H r\right)$ (thick black line) and the inverse function of the nonlinearity (colored curves in $A$ ). Spontaneous firing rates for the different nonlinearities are indicated by colored circles, for $h_{0}=0.30 \mathrm{~ms}^{-1}$. The addition of a static positive or negative stimulus (gray lines) shifts the operation point nonlinearly (circles plus error bars in the upper part of the figure). $C$ : Temporal evolution of the firing probability (top panel) and the feedback process (middle panel), under the effect of a particular time-dependent stimulus (bottom panel). In the absence of stimulus, $t<0 \mathrm{~ms}$, spontaneous firing rates are defined by the colored circles in $B$. The step stimulus deflects the firing probability transiently, until a new stationary value is reached. A periodic stimulus, $t>500 \mathrm{~ms}$, evokes a periodic response probability, whose amplitude is a non-linear function of the stimulus' amplitude. D: Spectral composition of filters' gain in the non-linear model. Continuous black line: gain of the feedback filter for the linear model, see Fig. 3A. Colors match the non-linearities shown in A. Symbols: ratio between the amplitudes of the sinusoidal response and stimulus, for a small sinusoidal stimulation, as a function of the driving frequency, $(1 / \sqrt{2 \pi}) \Delta r / \Delta s$. Irregular lines: gains of the Fourier transform of the filters obtained from the spike-triggered average of the nonlinear model, equivalent to $\hat{\chi}^{\mathrm{fb}}(\omega)$ for small stimuli, see Eq. (35). Dashed lines: equivalent linear filter of the non-linear model $\hat{h}^{\mathrm{nl}, \mathrm{fb}}(\omega)$, which can be approximated by an appropriate scaling of the linear filter, see Eq. (37). Parameters are identical to those used before for the linear model (see Figs. 2 and 3 ). 
steeper (blue line in Fig. 9 C), the firing probability evolves faster, and the initial transient becomes stronger.

The next step is to determine how time-dependent stimuli are processed. When a sinusoidal signal is applied ( $t>500 \mathrm{~ms}$, in Fig. 9 $\mathrm{C}$ ), the response probability is periodic, but not necessarily sinusoidal. Only in the limit of small $s(t)$ is the sinusoidal response guaranteed, and in this limit, the ratio between the (mean-subtracted) relative amplitudes of the input/output sinusoidal modulations is independent of the amplitude of the stimulus. The amplitude of the response, however, depends on the steepness of the nonlinearity (Fig. 9D).

To understand the spectral processing of the non-linear model, we define the transformed firing rate

$$
q(t)=f^{-1}[r(t)]
$$

so that Eq. (26) becomes

$$
q(t)=r_{0}+\int_{-\infty}^{\infty} h(\tau)\left[s_{1}(t-\tau)-g x(t-\tau)\right] \mathrm{d} \tau .
$$

In terms of $q(t)$, the feedback current $x(t)$, previously described by Eq. (8) , is now governed by

$$
\frac{\mathrm{d} x}{\mathrm{~d} t}=-\frac{x}{\tau_{\mathrm{d}}}+f[q(t)]
$$

Equations (29) and (30) constitute a closed system, but the presence of the nonlinearity precludes the application of the linear Fourier approach that allowed us, in the previous section, to find the relation between $h(\tau)$ and $h^{\mathrm{fb}}(\tau)$. Linearizing Eq. (30) around the operation point, $q_{0}=f^{-1}\left(r_{0}^{\mathrm{nl}}\right)$, yields

$$
\frac{\mathrm{d} x}{\mathrm{~d} t} \approx-\frac{x}{\tau_{\mathrm{d}}}+r_{0}^{\mathrm{nl}}+f^{\prime}\left(q_{0}\right)\left[q(t)-q_{0}\right]
$$

Equations (29) and (31) are closed and linear, and therefore, allow for a linear treatment. Transforming them both to Fourier space, and after some algebraic manipulations, 


$$
\begin{aligned}
\hat{q}(\omega)= & \sqrt{2 \pi}\left[q_{0} \delta(\omega)\right. \\
& \left.+\frac{\left(1+i \omega \tau_{\mathrm{d}}\right) \hat{h}(\omega)}{1+i \omega \tau_{\mathrm{d}}+\sqrt{2 \pi} g \tau_{\mathrm{d}} f^{\prime}\left(q_{0}\right) \hat{h}(\omega)} \hat{s}_{1}(\omega)\right] .
\end{aligned}
$$

Equation (32) can be written in terms of an effective filter $\hat{\chi}^{\mathrm{fb}}(\omega)$, such that

$$
\hat{q}(\omega)=\sqrt{2 \pi}\left[q_{0} \delta(\omega)+\hat{\chi}^{\mathrm{fb}}(\omega) \hat{s}_{1}(\omega)\right]
$$

where

$$
\hat{\chi}^{\mathrm{fb}}(\omega)=\frac{\left(1+i \omega \tau_{\mathrm{d}}\right) \hat{h}(\omega)}{1+i \omega \tau_{\mathrm{d}}+\sqrt{2 \pi} g \tau_{\mathrm{d}} f^{\prime}\left(q_{0}\right) \hat{h}(\omega)} .
$$

The possibility of summarizing the effect of feedback in Eq. (33) implies that in the temporal domain,

$$
r(t)=f\left[q_{0}+\int_{-\infty}^{+\infty} \chi^{\mathrm{fb}}(\tau) s_{1}(t-\tau) \mathrm{d} \tau\right] .
$$

The presence of the nonlinearity implies that the effective filter $\hat{\chi}^{\mathrm{fb}}(\omega)$ can no longer be calculated as the ratio between $\hat{r}(\omega)$ and $\hat{s}_{1}(\omega)$. Reverse correlation, however, still allow us to calculate $\chi^{\mathrm{fb}}(\tau)$ from the spike triggered average of the recorded data [Gabbiani and Koch 1998, Chichilnisky 2001]. The result is illustrated by the irregular lines in Fig. $9 D$.

Reverse correlation provides the best possible estimate of $\hat{\chi}^{\mathrm{fb}}(\omega)$, since the approach only entails the linearization of Eq. (8) . The method, however, requires large amounts of data to converge to a reliable estimation $\left(2.5 \times 10^{6}\right.$ spikes, in Fig. 9D). For practical purposes, hence, one may be willing to sacrifice some modeling accuracy, and further linearize Eq. (35), for the sake of obtaining an easier estimation method. Such approximation brings the response and the stimulus to be linearly related

$$
r(t) \approx f\left(q_{0}\right)+f^{\prime}\left(q_{0}\right) \int_{-\infty}^{+\infty} \chi^{\mathrm{fb}}(\tau) s_{1}(t-\tau) \mathrm{d} \tau,
$$

so that the linear response function of the non-linear system is 


$$
\hat{h}^{\mathrm{nl}, \mathrm{fb}}(\omega)=\frac{\left(1+i \omega \tau_{\mathrm{d}}\right) f^{\prime}\left(q_{0}\right)}{1+i \omega \tau_{\mathrm{d}}+\sqrt{2 \pi} g \tau_{\mathrm{d}} f^{\prime}\left(q_{0}\right) \hat{h}(\omega)} \hat{h}(\omega),
$$

differing from the linear approach $($ Eq. (13) $)$ by a rescaling controlled by the gain at the operation point.

In Fig. 9D, the spectral behavior of the filter obtained from the spike-triggered average of the non-linear model, $\hat{\chi}^{\mathrm{fb}}(\omega)$, as well as the linearized filter $\hat{h}^{\mathrm{nl}, \mathrm{fb}}(\omega)$ are shown. The symbols represent the results obtained from the ratio between $\hat{r}(\omega)$ and $\hat{s}_{1}(\omega)$, for small sinusoidal stimuli (defined as in Fig 7D). As observed, both filters are in excellent agreement with the simulated data. Clearly, as the non-linearity becomes steeper, the band-pass characteristics are more pronounced (see also, temporal responses in Fig. 9C).

The processing differences so far described for nonlinearities of varying steepness are also observed in a single nonlinearity, at varying operation points. In Fig. $10 A$, the operation point is varied by manipulating the value of $h_{0}$. The stationary firing rates are represented in Fig. 10B by symbols located on the nonlinearity (thick black line), whereas the gains are indicated on the derivative (gray line). The comparison between the nonlinear model with the linear approximations is similar to the comparison of Fig. 9D.

In the purely linear case, we observed that in certain conditions, feedback could give rise to unstable dynamics. Resonances, understood as peaks in the power spectrum of the effective filter, are still possible in the nonlinear case. However, now the resonant peak cannot grow indefinitely, so unstable divergences are ruled out. The linear approximation of Eq. (31) has a limited range of validity. For sigmoidal nonlinearities, as the firing rate increases, the operation point $r_{0}^{\mathrm{nl}}$ shifts upwards, so the slope $f^{\prime}\left(q_{0}\right)$ should not be taken as fixed. The value of the derivative diminishes progressively, as the flat part of the nonlinearity is approached. In Eq. (34), a diminished $f^{\prime}\left(q_{0}\right)$ is equivalent to a smaller feedback coupling constant $g$, thereby precluding divergences. Hence, although the firing probability can still contain a strong oscillatory component in the nonlinear case, the increased firing at the peaks of the oscillations acts as a self-regulatory mechanism, that forestalls unstable dynamics.

In the present section, we have introduced a nonlinearity in the relationship between $r(t)$ and $s_{1}(t)$. The feedback process, however, always remained linear. One could then wonder what is the effect of maintaining a linear relation between $r(t)$ and $s_{1}(t)$, but introducing a nonlinearity in the feedback process. From Eqs. (29) and (30), we see that in terms of $q(t)$, 

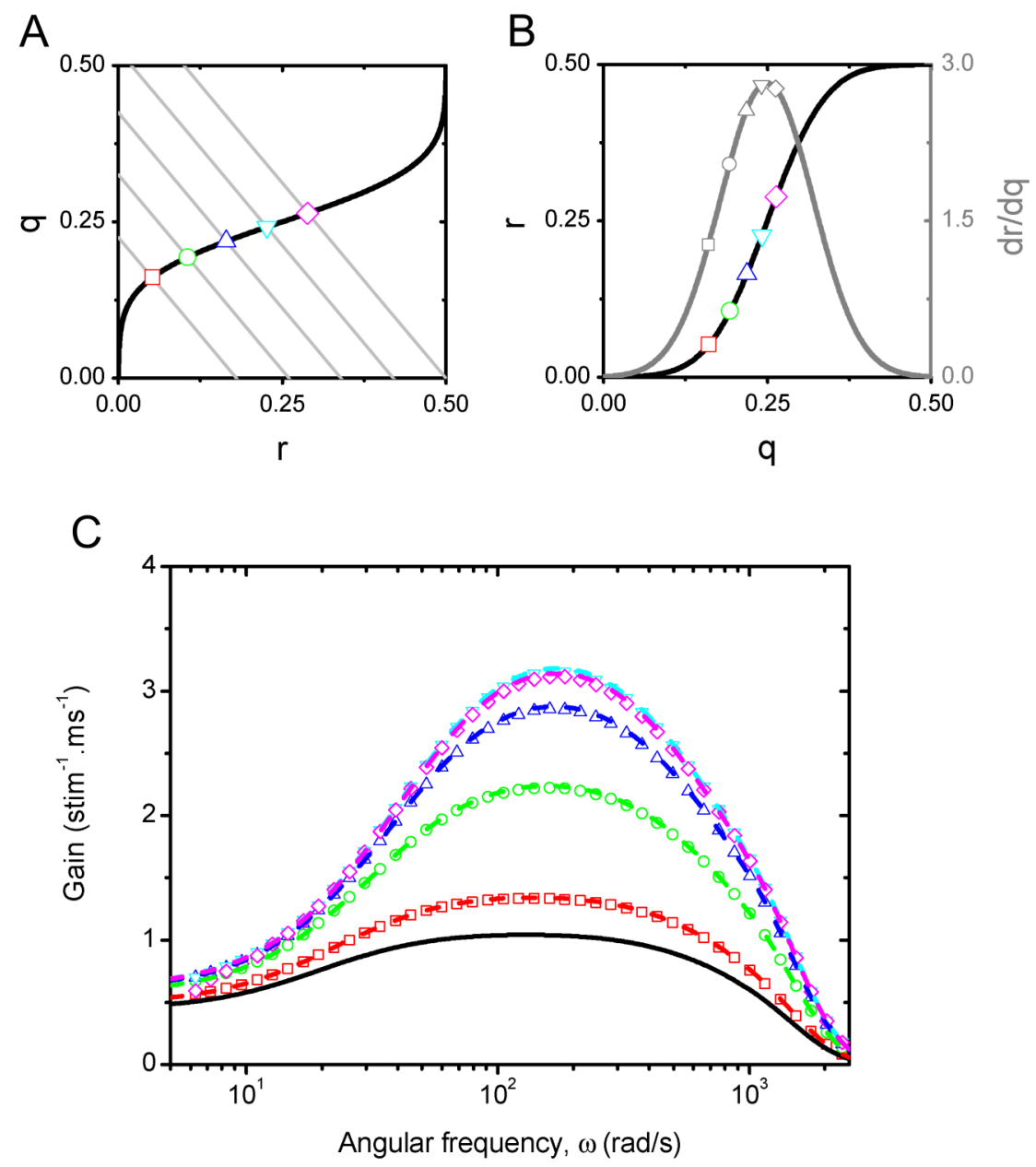

FIG. 10: Influence of the operation point in the nonlinear feedback model. A: A sigmoidal nonlinearity operated at different points. Different straight lines correspond to different values of $h_{0}\left(0.1,0.2,0.3,0.4\right.$ y $0.5 \mathrm{~ms}^{-1}$, respectively). The $y$-intercept is $h_{0}+H s_{0}$, with $H=$ $2.506(\mathrm{stim})^{-1} \cdot(\mathrm{ms})^{-1}$ and $s_{0}=0.05$ stimulus units, and the nonlinearity is defined as in Fig. 9A, with $\Delta=0.10$. $B$ : Firing probabilities, for the different values of $h_{0}$ defined in $A$, indicated by symbols located on the nonlinearity (thick black line, scale on the left margin). Associated gains are represented with corresponding symbols on the derivative of the function (gray line, scale on the right margin). C: Ratio between relative amplitudes of response and stimulus signals, for different driving frequencies. Symbols and colors correspond to those represented in A. Black thick line displays the spectral behavior of the lineal model, whereas colored dashed lines are appropriately scaled versions.

a nonlinear relation between $r(t)$ and $s_{1}(t)$ traduces into a nonlinear system of equations, where the nonlinearity appears in the feedback equation. Conversely, a nonlinearity in the feedback process that modifies the second term of the right-hand side of Eq. (8) can be treated, from the mathematical point of view, in the same way as the the nonlinear system explored in this section. More general nonlinear feedback processes, for example, modifying 
the first term of the right-hand side of Eq. (8), or even mixing the two terms together, require additional techniques.

\section{Higher-dimensional receptive fields}

The results obtained so far remain unchanged if the stimulus (and therefore also the linear filter) depends on several dimensions, and not just time, as long as adaptation is homogeneous in those dimensions. In this case, we write the external stimulus as $s(\mathbf{y}, t)$, where $\mathbf{y} \in \mathcal{D}$ is a vector in a one- or a multi-dimensional space $\mathcal{D}$ representing the relevant features of the sensory modality under study (direction of the incoming light or chromatic composition in the case of vision, frequency content in the case of audition, etc). The external stimulus is filtered by some sort of transduction process and perhaps also by one or more neurons that lie between the sensory receptors and the neuron under study. We represent such upstream filtering processes by means of a high-dimensional filter $h_{u}(\mathbf{y}, \tau)$, that transforms the high-dimensional stimulus $s(\mathbf{y}, t)$ in a purely temporal ionic input current $s_{1}(t)$,

$$
s_{1}(t)=\int_{\mathcal{D}} \int_{-\infty}^{+\infty} h_{u}(\mathbf{y}, \tau) s(\mathbf{y}, t-\tau) \mathrm{d} \mathbf{y} \mathrm{d} \tau .
$$

This expression can be inserted into Eq. (2) to construct the new full input current $I(t)$ needed in Eq. (1). Notice that in the present framework, the high-dimensional stimulus is first filtered in the $\mathbf{y}$-dimensional and temporal domains by $h_{u}(\mathbf{y}, \tau)$ (Eq. (38)), and afterwards by the temporal filter $h(\tau)$ (Eq. (1i)). Using a Fourier analysis completely analogous to the one developed before, we arrive at

$$
\begin{array}{r}
\hat{r}(\omega)=\frac{\sqrt{2 \pi}\left(1+i \omega \tau_{\mathrm{d}}\right)}{1+i \omega \tau_{\mathrm{d}}+\sqrt{2 \pi} g \tau_{\mathrm{d}} \hat{h}(\omega)}\left[r_{0} \delta(\omega)\right. \\
\left.+\sqrt{2 \pi} \hat{h}(\omega) \int_{\mathcal{D}} \hat{h_{u}}(\mathbf{y}, \omega) \hat{s}(\mathbf{y}, \omega) \mathrm{d} \mathbf{y}\right] .
\end{array}
$$

This expression reduces to Eq. (9) when $h_{u}(\mathbf{y}, \tau)=\delta\left(\mathbf{y}-\mathbf{y}_{0}\right) \delta(\tau)$.

By stimulating the cell with signals that are localized in the additional dimensions, $s(\mathbf{y}, t)=\delta\left(\mathbf{y}-\mathbf{y}_{0}\right) f(t)$, the whole of the previous theory becomes valid for each chosen $\mathbf{y}_{0}$. In particular, feedback still transforms the intrinsic receptive field in an effective 
receptive field by multiplying the Fourier transform of the former by a factor that depends on the frequency, but does not depend on the stimulation point $\mathbf{y}_{0}$. At least, such is the effect of feedback if we may assume that the signal $x(t)$ only depends on the output of the cell and is not modulated, for example, by spatial input components.

\section{DISCUSSION AND CONCLUSIONS}

In this paper, we analyzed how spike-evoked negative feedback modifies the effective receptive field of a cell. The approach was based on an ideal concept, here named perfect feedback, where the signal $x(t)$ is not a function of the actual spikes generated by the neuron, but rather of the probability that spikes be generated. This assumption is ultimately unrealistic, but becomes a good approximation of the real system when (a) the stimulus varies slowly compared to the inter-spike interval of the neuron under study, or (b) feedback is mediated by a large number of similar neurons in the network. In both cases, the firing probability $r(t)$ is sampled exhaustively, and therefore, the distribution of sampled signals follows the probability $r(t)$ closely during the time scale $\tau_{\mathrm{d}}$ governing the feedback process. This idealized scenario allowed us to develop an analytical approach, and to derive the mathematical connection between the intrinsic filter $h(\tau)$ and the effective filter $h^{\mathrm{fb}}(\tau)$. In particular, Eq. (13) provides the link by which intrinsic or feedback parameters shape the receptive field.

Previous studies have reported some reshaping of receptive fields as the input changes. For example, in the case of visual stimuli, the spatial and the temporal context alter the input/output transformation, as the system adapts to the local statistics [Schwartz et al. 2007, Wark et al. 2007, Wark et al. 2009], producing changes all the way up to the perceptual level [Schwartz et al. 2007, Kohn 2007, Lochmann et al. 2012]. In single cells, both the total luminance and the amount of contrast shape linear filters with increased band-pass characteristics [Enroth-Cugell and Shapley 1973, Kaplan and Benardete 2001]. The same effects are observed in single auditory cells, as the mean and the variance of synthetic sounds are manipulated [Nagel and Doupe 2006]. According to our results, these phenomena could be explained by modulating the amount of feedback as the signal varies. The modulation could be mediated by synaptic scaling, in the case of network-based feedback processes, or by ionic mechanisms, in the case of single cell adaptation, eg. by $\mathrm{Ca}^{2+}$ concentration. Moreover, the 
reshaping of filters can also be modulated by the spatial structure of the stimulus. For example, the receptive fields processing stimuli with natural spatio-temporal statistics are different from those obtained from simple ensembles. When the higher-order input statistics are taken into account in the estimation of receptive fields [Theunissen et al. 2001], the temporal profile of the filters processing natural stimuli turn out to be biphasic, whereas monophasic filters are obtained in responses to gratings [David et al. 2004]. In terms of our analysis, this change could be explained if the amount of negative feedback depended on the statistical properties of the input signal. However, given the different long-range behavior of spatial correlations for different ensembles, this would require to extend the model in order to include some spatial dependence of the feedback signal, for example, associated to the spiking activity of neurons that respond to stimuli presented in a shifted position.

In the purely linear case, positive feedback always produces unstable dynamics. If an ON cell is subject to positive feedback, any positive stimulus fluctuation, no matter how small, feeds a reverberating loop where activity eventually diverges. Any negative stimulus fluctuation, in turn, eventually extinguishes firing altogether. An OFF cell is also unstable, with the opposite effect of positive and negative stimulus fluctuations. Such unstable systems are all-or-none (divergence or extinction) and have therefore not been studied here.

An important finding of this paper is that negative feedback, which is usually assumed to exert a regularizing effect, can also produce unstable dynamics in the linear case. Negative feedback always diminishes the responses to slow stimuli, enhancing the band-pass characteristics of the filtering process. However, in certain conditions, these characteristics can be magnified dramatically, up to the point that the effective filter $\hat{h}^{\mathrm{fb}}(\omega)$ be sharply peaked at a specific frequency $\omega_{0}$. In the time domain, $h^{\mathrm{fb}}(\tau)$ exhibits pronounced oscillations at this particular frequency. Feedback, hence, transformed an intrinsic receptive field that acted as an integrator into an effective resonator [Mato and Samengo 2008].

Neurons often display resonant properties. A widely accepted view states that oscillatory properties can either stem from intrinsic cellular characteristics or from network interactions [Buzsáki 2006, Prescott et al. 2008, Wang 2010]. The important ingredient is that two types of mechanisms coexist [Hutcheon and Yarom 2000]: those attenuating high frequencies (typically, leak currents), and those attenuating low frequencies. Many processes can be invoked to attenuate low frequencies, both at the single-cell (e. g., leak filtering), and the network level (e. g., synaptic filtering). Previous studies of resonant behavior 
have attempted to understand resonances in terms of such properties, invoking particular subthreshold processes Izhikevich 2007, Gutfreund et al. 1995, Hutcheon et al. 1996a, Hutcheon et al. 1996b, Richardson et al. 2003], or specific network interactions [Wang 2010, Lampl and Yarom 1997, Köndgen et al. 2008, Buzsáki and Wang 2012]. In addition, to avoid falling into quiescence, amplifying processes are sometimes also invoked. In agreement with previous studies on properties of neurons with spike-frequency adaptation Benda and Herz 2003, Gigante et al. 2007, Benda and Hennig 2008, Benda et al. 2010], in this paper we have shown that negative feedback suffices to attenuate low frequencies. We make no assumptions about subthreshold properties, inasmuch as they produce an intrinsic receptive field that contains a maximal cutoff frequency. In addition, no ad-hoc mechanisms are required to produce amplification, as long as the baseline firing rate $r_{0}^{\mathrm{fb}}$ remains positive, for which a strong stimulus baseline $s_{0}$, or a strong spontaneous rate $h_{0}$, suffice. Moreover, the formalism proposed here is general enough as to be equally applicable to networkmediated feedback currents, or to intrinsic adaptation currents. Our idealized approach, hence, provides a unified description of the mechanisms through which spike-triggered negative feedback induces resonances.

In order to test the validity of the idealized approach provided by perfect feedback, we also ran numeric simulations where a finite number $N$ of individual feedback signals $x_{i}(t)$ were triggered by actual spikes. Importantly, we concluded that the effective filtering characteristics do not depend on $N$. Perfect feedback, hence, can be safely used to study both self-inhibition $(N=1)$ and network-induced regulation $(N>1)$. The analytic study of the fluctuations in the feedback signal and in the response, however, shows that the amount of noise in the output spectrum diminishes as $1 / N$ (Eqs. (22) and (25)). Moreover, the shape of the noise spectrum is given by a Cauchy distribution [Johnson et al. 1994].

The above conclusions hold for linear Poisson neuron models. Only for the linear case can the analytic approach be developed. The concept of receptive field, however, can also be extended to the case of linear-nonlinear Poisson models, or generalized nonlinear models. Although, strictly speaking, the presence of a nonlinearity does not allow us to employ linear methods, in the last section of this paper we demonstrated that by linearizing one of the two equations governing the system, an approximate description of the nonlinear case is possible. We showed that feedback still produces an effective receptive field that is narrower than the intrinsic one, and that resonances may also appear. Moreover, the nonlinear description is 
also useful to understand how the divergences obtained in the purely linear case saturate at a finite value in the nonlinear description.

\section{Acknowledgments}

This work has been funded by Consejo Nacional de Investigaciones Científicas y Técnicas, Agencia Nacional de Promoción Científica y Tecnológica, Universidad Nacional de Cuyo and Comisión Nacional de Energía Atómica, all from República Argentina.

\section{Appendix A: Appendix}

The convention used here to operate with the Fourier transform is

$$
\hat{f}(\omega)=\frac{1}{\sqrt{2 \pi}} \int_{-\infty}^{+\infty} f(t) \mathrm{e}^{-i \omega t} \mathrm{~d} t .
$$

From this definition, the following properties follow:

- The Fourier transform of a constant signal of magnitude $r_{0}$ is $\hat{f}(\omega)=\sqrt{2 \pi} r_{0} \delta(\omega)$.

- If a signal $f(t)$ is equal to the convolution of two other signals $g(t)$ and $h(t)$, then $\hat{f}(\omega)=\sqrt{2 \pi} \hat{g}(\omega) \hat{h}(\omega)$.

- If a signal $f(t)$ is equal to another signal but delayed, $f(t)=g(t-\Delta)$, then $\hat{f}(\omega)=$ $\mathrm{e}^{-i \omega \Delta} \hat{g}(\omega)$.

- If a signal $f(t)$ is the derivative of another signal $f(t)=\mathrm{d} g / \mathrm{d} t$, then $\hat{f}(\omega)=i \omega \hat{g}(\omega)$.

The Laplace transform, in turn, is defined as

$$
\tilde{f}(s)=\int_{0}^{\infty} f(t) \mathrm{e}^{-s t} \mathrm{~d} t
$$

where $s=\sigma+i \omega$ is a complex number. In the particular case where $s$ is evaluated at a purely imaginary number $(\sigma=0)$, the Laplace transform is proportional to the Fourier transform for temporally positive functions. The Laplace transform, hence, can be seen as a generalization of the Fourier transform to the whole complex plane. Related properties hold: 
- The Laplace transform of a constant signal of magnitude $r_{0}$ is $\tilde{f}(s)=r_{0} / s$.

- If a signal $f(t)$ is equal to the convolution of two other signals $g(t)$ and $h(t)$, then $\tilde{f}(s)=\tilde{g}(s) \tilde{h}(s)$.

- If a signal $f(t)$ is the derivative of another signal $f(t)=\mathrm{d} g / \mathrm{d} t$, then $\tilde{f}(s)=s \tilde{g}(s)-$ $g(0)$.

Using these properties, it is easy to see that if the signals $r(t)$ and $x(t)$ are governed by Eqs. (4) and (8), the Laplace transform of $r(t)$, for an initial zero feedback contribution $x(0)=0$, is

$$
\tilde{r}(s)=\frac{r_{0} / s+\tilde{h}(s) \tilde{s}_{1}(s)}{1+g \tilde{h}(s) /\left(s+1 / \tau_{\mathrm{d}}\right)} .
$$

According to control theory [Franklin et al. 1994], this feedback system becomes unstable when at least one pole of $\tilde{r}(s)$ has positive real part. It is important to search for instabilities in the Laplace representation, since they may not be evident in the Fourier space. In addition to the fixed pole $s=0$ given by the constant signal term, the poles of $\tilde{r}(s)$ are those complex points $s$ where the denominator of Eq. (A3) vanish, that is,

$$
1+g \frac{\tilde{h}(s)}{s+1 / \tau_{\mathrm{d}}}=0 .
$$

This equation holds in the complex plane, so both the real and the imaginary part of the equality must vanish. Feedback gives rise to unstable behavior whenever at least one solution of Eq. (A4) has positive real part. The onset of instability, hence, appears when the pole (or pair of conjugate poles) with largest real part crosses the imaginary axis, from left to right. At the crossing, $\sigma=0$, so the real and imaginary parts of Eq. (A4) become

$$
\begin{aligned}
\operatorname{Im}[\hat{h}(\omega)]-\tau_{\mathrm{d}} \omega \operatorname{Re}[\hat{h}(\omega)] & =0, \\
1+\sqrt{2 \pi} g \tau_{\mathrm{d}} \operatorname{Re}[\hat{h}(\omega)] & =0,
\end{aligned}
$$

where now, $\hat{h}(\omega)$ is the Fourier transform of the intrinsic filter, obtained when evaluating the Laplace transform at a purely imaginary point and dividing by $\sqrt{2 \pi}$. The frequencies satisfying Eq. (A5) are independent of the feedback strength, $g$. Note that, in addition, Eq. (A6) can only be fulfilled by those frequencies $\omega_{i}$, from the set of solutions to Eq. (A5), 
that result in $\operatorname{Re}\left[\hat{h}\left(\omega_{i}\right)\right]<0$. Even among these, if $g$ is small, no frequency $\omega$ satisfies Eq. ( $(\mathrm{A} 6)$, and the system is stable (the pole with largest real part is on the stable semiplane). For a critical value of feedback strength, the condition imposed by Eq. (A6) can be finally reached and the system becomes unstable. This means that for feedback strengths beyond the critical value, there is at least one pole on the unstable semi-plane and therefore the inverse Fourier transform diverges.

\section{Appendix B: Appendix}

In this section, we describe the temporal processing of slow stimuli. We arrive at the same results as the ones derived by [Urdapilleta and Samengo 2009], but here we base the analysis on the spectral properties of filters. In the absence of feedback,

$$
\begin{aligned}
& r(t)=h_{0}+\int_{-\infty}^{+\infty} h(\tau) s(t-\tau) \mathrm{d} \tau \\
& \hat{r}(\omega)=\sqrt{2 \pi}\left[h_{0} \delta(\omega)+\hat{h}(\omega) \hat{s}(\omega)\right] .
\end{aligned}
$$

In the present context, a slow stimulus is one that does not contain high frequency components. In other words, the input/output relation of the cell is only determined by the lowest frequency components of the filter $\hat{h}(\omega)$, since the higher frequencies are not explored. The stimulus, hence, has to remain fairly constant throughout the time scale of the filter (given by the non-zero portions in the temporal filters shown in Fig. 1). If only low frequencies matter, we may take the limit $\omega \rightarrow 0$. In this context, we prove that

- ON cells behave as low-pass filters, see Fig. 1A. For $\omega \rightarrow 0$, the Fourier transform of these filters is

$$
\begin{aligned}
\hat{h}(\omega) & =\frac{1}{\sqrt{2 \pi}} \int_{-\infty}^{+\infty} h(\tau) \mathrm{e}^{-i \omega \tau} \mathrm{d} \tau \\
& =\frac{1}{\sqrt{2 \pi}} \int_{-\infty}^{+\infty} h(\tau)[\cos (\omega \tau)-i \sin (\omega \tau)] \mathrm{d} \tau \\
& \approx \frac{1}{\sqrt{2 \pi}} \int_{-\infty}^{+\infty} h(\tau)[1-i \omega \tau] \mathrm{d} \tau=\frac{1}{\sqrt{2 \pi}}\left(H+i \omega H_{1}\right)
\end{aligned}
$$


where $H=\int_{-\infty}^{+\infty} h(\tau) \mathrm{d} \tau$ and $H_{1}=-\int_{-\infty}^{+\infty} \tau h(\tau) \mathrm{d} \tau$. The small angle approximation can be used again,

$$
\begin{aligned}
\hat{h}(\omega) & \approx \frac{H}{\sqrt{2 \pi}}\left[1+i \omega \frac{H_{1}}{H}\right] \\
& \approx \frac{H}{\sqrt{2 \pi}}\left[\cos \left(\omega \frac{H_{1}}{H}\right)+i \sin \left(\omega \frac{H_{1}}{H}\right)\right] \\
& \approx \frac{H}{\sqrt{2 \pi}} \mathrm{e}^{-i \omega \delta_{0}},
\end{aligned}
$$

where we have defined the (positive) delay $\delta_{0}=-H_{1} / H$. From this expression, it is easy to check that the gain of the Fourier transform for ON cells at low frequencies is constant and equal to $H / \sqrt{2 \pi}$. In addition, the phase decreases linearly with $\omega$, starting at 0 with slope $-\delta_{0}$ (in Fig. 17A, a linear graph instead of the semi-logarithmic one would clearly show this linear relationship).

To relate $\hat{h}(\omega)$ to the neural response in the temporal domain, we simply make use of the Fourier transform of a delayed signal (see Appendix $\mathrm{A}$ ),

$$
r(t) \approx h_{0}+\mathcal{F}^{-1}\left\{H \mathrm{e}^{-i \omega \delta_{0}} \hat{s}(\omega)\right\} \approx h_{0}+H s\left(t-\delta_{0}\right) .
$$

According to Eq. (B4), the amplitude of the response is exclusively determined by $H$; simultaneously, neural processing introduces a fixed delay $\delta_{0}$ between the response and the stimulus.

- ON biphasic cells behave as band-pass filters, see Fig. 1C. In the limit $\omega \rightarrow 0, \hat{h}(\omega)$ is still given by Eq. (B1). However, symmetric biphasic filters satisfy $H=0$, so to obtain a meaningful description we have to perform the expansion around $\omega=0$ up to the second order,

$$
\begin{aligned}
\hat{h}(\omega) & \approx \frac{1}{\sqrt{2 \pi}} \int_{-\infty}^{+\infty} h(\tau)\left[1-i \omega \tau-\frac{1}{2} \omega^{2} \tau^{2}\right] \mathrm{d} \tau \\
& \approx \frac{1}{\sqrt{2 \pi}}\left(H+i \omega H_{1}-\frac{1}{2} \omega^{2} H_{2}\right) \\
& \approx \frac{i H_{1} \omega}{\sqrt{2 \pi}}\left[1+i \omega \frac{H_{2}}{2 H_{1}}\right] .
\end{aligned}
$$


where $H_{2}=\int_{-\infty}^{+\infty} \tau^{2} h(\tau) \mathrm{d} \tau$. Proceeding as before and defining a corresponding (positive) delay $\delta_{1}=-H_{2} /\left(2 H_{1}\right)$, we obtain

$$
\begin{aligned}
\hat{h}(\omega) & \approx \frac{H_{1} \omega}{\sqrt{2 \pi}} \mathrm{e}^{i \pi / 2}\left[\cos \left(\omega \frac{H_{2}}{2 H_{1}}\right)+i \sin \left(\omega \frac{H_{2}}{2 H_{1}}\right)\right] \\
& \approx \frac{H_{1} \omega}{\sqrt{2 \pi}} \mathrm{e}^{i \pi / 2} \mathrm{e}^{-i \omega \delta_{1}}=\frac{H_{1} \omega}{\sqrt{2 \pi}} \mathrm{e}^{i\left(\pi / 2-\omega \delta_{1}\right)} .
\end{aligned}
$$

Hence, in the limit $\omega \rightarrow 0$, the gain of symmetric biphasic filters depends linearly on the angular frequency, with a positive slope $H_{1} / \sqrt{2 \pi}$. In addition, the filter phase is a linearly decreasing function of $\omega$, with intercept at the origin $\pi / 2$ and slope $-\delta_{1}$. Both characteristics can be observed in Fig. 11C.

To relate $\hat{h}(\omega)$ to the neural response in the temporal domain, it is useful to recover the imaginary unit as a factor and rewrite Eq. (B6) as

$$
\hat{h}(\omega) \approx \frac{H_{1}}{\sqrt{2 \pi}} \mathrm{e}^{-i \omega \delta_{1}}(i \omega)
$$

In this case, given that the Fourier transform of a convolution becomes a product in Fourier space, the term $(i \omega)$ can be effectively associated to the stimulus, $\hat{s}(\omega)$. In turn, by applying the Fourier transform of a derivative, and proceeding as before, it is easy to check that $r(t)$ is proportional to the delayed stimulus derivative, with a factor of proportionality given by $H_{1}$ and a fixed delay $\delta_{1}$. Explicitly,

$$
r(t) \approx h_{0}+\mathcal{F}^{-1}\left\{H_{1} \mathrm{e}^{-i \omega \delta_{1}}[i \omega \hat{s}(\omega)]\right\} \approx h_{0}+H_{1}\left[\frac{\mathrm{d} s}{\mathrm{~d} t}\right]_{\left(t-\delta_{1}\right)} .
$$

- Corresponding OFF cells behave analogously to monophasic and biphasic ON cells (see Figs. 1B and 1D). Specifically, the Fourier transforms of these filters are exactly given by Eqs. (B2) and ( $(\overline{\mathrm{B} 5})$, in the limit $\omega \rightarrow 0$. The only difference with the previous ON cells is that the proportionality factors $H$ and $H_{1}$ for monophasic and biphasic cells, respectively, are now negative. The presence of a factor $(-1)$ affecting the whole expression can be incorporated into a multiplicative term, $\exp ( \pm i \pi)$, which shifts the phase of the Fourier transform in $\pm \pi$. This shift can be observed in Fig. 1 by 
comparing phases of corresponding filters (monophasic ON/OFF filters and biphasic $\mathrm{ON} / \mathrm{OFF}$ filters). Additionally, the factor $(-1)$ does not affect the magnitude of the Fourier transform of $\mathrm{ON}$ or $\mathrm{OFF}$ cells. In the temporal domain, this factor simply means that the relationships obtained for ON cells are also valid, but associated to the negative of the stimulus or its derivative. These results agree our previous analysis [Urdapilleta and Samengo 2009].

[Amit and Brunel 1997] Amit, D. J., \& Brunel, N. (1997). Model of global spontaneous activity and local structured activity during delay periods in the cerebral cortex. Cerebral Cortex, 7 , $237-252$.

[Atwood and Karunanithi 2002] Atwood, H. L., \& Karunanithi, S. (2002). Diversification of synaptic strength: Presynaptic elements. Nature Reviews Neuroscience, 3, 497-516.

[Ayaz and Chance 2009] Ayaz, A., \& Chance, F. S. (2009). Gain modulation of neuronal responses by subtractive and divisive mechanisms of inhibition. Journal of Neurophysiology, 101, 958968.

[Baccus and Meister 2002] Baccus S. A., \& Meister, M. (2002). Fast and slow contrast adaptation in retinal circuitry. Neuron, 36, 909-919.

[Benda and Hennig 2008] Benda, J., \& Hennig, R. M. (2008). Spike-frequency adaptation generates intensity invariance in a primary auditory interneuron. Journal Computational Neuroscience, 24, 113-136.

[Benda and Herz 2003] Benda, J., \& Herz, A. V. M. (2003). A universal model for spike-frequency adaptation. Neural Computation, 15, 2523-2564.

[Benda et al. 2010] Benda, J., Maler, L., \& Longtin, A. (2010). Linear versus nonlinear signal transmission in neuron models with adaptation currents or dynamic thresholds. Journal of Neurophysiology, 104, 2806-2820.

[Borst et al. 2005] Borst, A., Flanagin, V. L., \& Sompolinsky, H. (2005). Adaptation without parameter change: Dynamic gain control in motion detection. Proceedings of the National Academy of Sciences USA, 102(17), 6172-6176.

[Bressloff 2012] Bressloff, P. C. (2012). Spatiotemporal dynamics of continuum neural fields. Journal of Physics A: Mathematical and Theoretical, 45, 033001. 
[Buice et al. 2010] Buice, M. A., Cowan, J. D., \& Chow, C. C. (2010). Systematic fluctuation expansion for neural network activity equations. Neural Computation, 22, 377-426.

[Buonomano and Maass 2009] Buonomano, D. V., \& Maass, W. (2009). State-dependent computations: Spatiotemporal processing in cortical networks. Nature Reviews Neuroscience, 10, 113-125.

[Butts et al. 2011] Butts, D. A., Weng, C., Jin, J., Alonso, J.M., \& Paninski, L. (2011). Temporal precision in the visual pathway through the interplay of excitation and stimulus-driven suppression. Journal of Neuroscience, 31(31), 11313-11327.

[Buzsáki 2006] Buzsáki, G. (2006). Rhythms of the Brain. New York: Oxford University Press.

[Buzsáki and Wang 2012] Buzsáki, G., \& Wang, X. J. (2012). Mechanisms of gamma oscillations. Annual Review of Neuroscience, 35, 203-225.

[Carandini and Heeger 2012] Carandini, M., \& Heeger, D. J. (2012). Normalization as a canonical neural computation. Nature Reviews Neuroscience, 13, 51-62.

[Carandini et al. 1997] Carandini, M., Heeger, D. J., \& Movshon, J. A. (1997). Linearity and normalization in simple cells of the macaque primary visual cortex. Journal of Neuroscience, $17(21), 8621-8644$.

[Chichilnisky 2001] Chichilnisky, E. J. (2001). A simple white noise analysis of neuronal light responses. Network: Computation in Neural Systems, 12, 199-213.

[Coombes and Laing 2009] Coombes, S., \& Laing, C. (2009). Delays in activity-based neural networks. Philosophical Transactions of the Royal Society A: Mathematical, Physical and Engineering Sciences, 367, 1117-1129.

[David and Friston 2003] David, O., \& Friston, K. J. (2003). A neural mass model for MEG/EEG: Coupling and neuronal dynamics. NeuroImage, 20, 1743-1755.

[David et al. 2004] David, S. V., Vinje, W. E., \& Gallant, J. L. (2004). Natural stimulus statistics alter the receptive field structure of V1 neurons. Journal of Neuroscience, 24(31), 6991-7006.

[Dayan and Abbott 2001] Dayan, P., \& Abbott, L. F. (2001). Theoretical Neuroscience: Computational and Mathematical Modeling of Neural Systems. Cambridge: The MIT Press.

[Dong and Atick 1995] Dong, D. W., \& Atick, J. J. (1995). Statistics of natural time-varying images. Network: Computation in Neural Systems, 6(3), 345-358.

[Douglas et al. 1995] Douglas, R. J., Koch, C., Mahowald, M., Martin, K. A. C., \& Suarez, H. H. (1995). Recurrent excitation in neocortical circuits. Science, 269, 981-985. 
[Enroth-Cugell and Shapley 1973] Enroth-Cugell, C., \& Shapley, R. M. (1973). Adaptation and dynamics of cat retinal ganglion cells. Journal of Physiology, 233, 271-309.

[Eytan et al. 2003] Eytan, D., Brenner, N., \& Marom, S. (2003). Selective adaptation in networks of cortical neurons. Journal of Neuroscience, 23(28), 9349-9356.

[Feldman 2009] Feldman, D. E. (2009). Synaptic mechanisms for plasticity in neocortex. Annual Review of Neuroscience, 32, 33-55.

[Felsen et al. 2002] Felsen, G., Shen, Y. S., Yao, H., Spor, G., Li, C., \& Dan, Y. (2002). Dynamic modification of cortical orientation tuning mediated by recurrent connections. Neuron, 36, 945-954.

[Franklin et al. 1994] Franklin, G. F., Powell, J. D., \& Emami-Naeini, A. (1994). Feedback Control of Dynamic Systems. Reading, MA: Addison-Wesley, 3rd edition.

[Freeman 1972a] Freeman, W. J. (1972a). Measurement of open-loop responses to electrical stimulation in olfactory bulb of cat. Journal of Neurophysiology, 35(6), 745-761.

[Freeman 1972b] Freeman, W. J. (1972b). Measurement of oscillatory responses to electrical stimulation in olfactory bulb of cat. Journal of Neurophysiology, 35(6), 762-779.

[Freeman 1972c] Freeman, W. J. (1972c). Linear analysis of the dynamics of neural masses. Annual Review of Biophysics and Bioengineering, 1, 225-256.

[Freeman 1987] Freeman, W. J. (1987). Simulation of chaotic EEG patterns with a dynamic model of the olfactory system. Biological Cybernetics, 56, 139-150.

[Gabbiani and Koch 1998] Gabbiani, F., \& Koch, C. (1998). Principles of spike train analysis. In C. Koch \& I. Segev (Eds.), Methods in Neuronal Modeling: From Ions to Networks. Cambridge MA: MIT Press.

[Gardiner 1985] Gardiner, C. W. (1985). Handbook of Stochastic Methods: For Physics, Chemistry and the Natural Sciences. Berlin: Springer-Verlag.

[Garvert and Gollisch 2013] Garvert, M. M., \& Gollisch, T. (2013). Local and global contrast adaptation in retinal ganglion cells. Neuron, 77, 915-928.

[Gaudry and Reinagel 2007] Gaudry, K. S., \& Reinagel, P. (2007). Contrast adaptation in a nonadapting LGN model. Journal of Neurophysiology, 98, 1287-1296.

[Geisler 2008] Geisler, W. S. (2008). Visual perception and the statistical properties of natural scenes. Annual Review of Psychology, 59, 167-192.

[Gigante et al. 2007] Gigante, G., Del Giudice, P., \& Mattia, M. (2007). Frequency-depdendent 
response properties of adapting spiking neurons. Mathematical Biosciences, 207(2), 336-351.

[Gutfreund et al. 1995] Gutfreund, Y., Yarom, Y., \& Segev, I. (1995). Subthreshold oscillations and resonant frequency in guinea-pig cortical neurons: physiology and modelling. Journal of Physiology, 483(3), 621-640.

[Heeger 1992] Heeger, D. J. (1992). Normalization of cell responses in cat striate cortex. Visual Neuroscience, 9, 181-197.

[Hutcheon et al. 1996a] Hutcheon, B., Miura, R. M., \& Puil, E. (1996). Subthreshold membrane resonance in neocortical neurons. Journal of Neurophysiology, 76(2), 683-697.

[Hutcheon et al. 1996b] Hutcheon, B., Miura, R. M., \& Puil, E. (1996). Models of subthreshold membrane resonance in neocortical neurons. Journal of Neurophysiology, 76(2), 698-714.

[Hutcheon and Yarom 2000] Hutcheon, B., \& Yarom, Y. (2000). Resonance, oscillation and the intrinsic frequency preferences of neurons. Trends in Neurosciences, 23(5), 216-222.

[Izhikevich 2007] Izhikevich, E. M. (2007). Dynamical Systems in Neuroscience: The Geometry of Excitability and Bursting. Cambridge, Massachussets: MIT Press.

[Johnson et al. 1994] Johnson, N. L., Kotz, S., \& Balakrishnan, N. (1994). Continuous Univariate Distributions, 2nd ed.. New York: Wiley.

[Kaplan and Benardete 2001] Kaplan, E., \& Benardete, E. (2001). The dynamics of primate retinal ganglion cells. Progress in Brain Research, 134, 17-34.

[Kohn 2007] Kohn, A. (2007). Visual adaptation: Physiology, mechanisms, and functional benefits. Journal of neurophysiology, 97, 3155-3164.

[Köndgen et al. 2008] Köndgen, H., Geisler, C., Fusi, S., Wang, X. J., Lüscher, H. R., \& Giugliano, M. (2008). The dynamical response properties of neocortical neurons to temporally modulated noisy inputs in vitro. Cerebral Cortex, 18, 2086-2097.

[Ladenbauer et al. 2014] Ladenbauer, J., Augustin, M., \& Obermayer, K. (2014). How adaptation currents change threshold, gain, and variability of neuronal spiking. Journal of Neurophysiology, 111, 939-953.

[Lampl and Yarom 1997] Lampl, I., \& Yarom, Y. (1997). Subthreshold oscillations and resonant behavior: Two manifestations of the same mechanism. Neuroscience, 78(2), 325-341.

[Ledoux and Brunel 2011] Ledoux, E., \& Brunel, N. (2011). Dynamics of networks of excitatory and inhibitory neurons in response to time-dependent inputs. Frontiers in Computational Neuroscience, 5(25), 1-17. 
[Lochmann et al. 2012] Lochmann, T., Ernst, U. A., \& Denève, S. (2012). Perceptual inference predicts contextual modulations of sensory responses. Journal of neuroscience, 32(12), 41794195 .

[Mante et al. 2005] Mante, V., Frazor, R. A., Bonin, V., Geisler, W. S., \& Carandini, M. (2005). Independence of luminance and contrast in natural scenes and in the early visual system. Nature Neuroscience, 8(12), 1690-1697.

[Mato and Samengo 2008] Mato, G., \& Samengo, I. (2008). Type I and type II neuron models are selectively driven by differential stimulus features. Neural Computation, 20, 2418-2440.

[Nagel and Doupe 2006] Nagel, K. I., \& Doupe, A. J. (2006). Temporal processing and adaptation in the songbird auditory forebrain. Neuron, 51, 845-859.

[Peron and Gabbiani 2009] Peron, S., \& Gabbiani, F. (2009). Spike-frequency adaptation mediates looming stimulus selectivity in a collision-detecting neuron. Nature Neuroscience, 12(3), 318326.

[Prescott et al. 2008] Prescott, S. A., Ratté, S., De Koninck, Y., \& Sejnowski, T. J. (2008). Pyramidal neurons switch from integrators in vitro to resonators under in vivo-like conditions. Journal of Neurophysiology, 100, 3030-3042.

[Prescott and Sejnowski 2008] Prescott, S. A., \& Sejnowski, T. J. (2008). Spike-rate coding and spike-time coding are affected oppositely by different adaptation mechanisms. Journal of Neuroscience, 28(50), 13649-13661.

[Press et al. 2007] Press, W. H., Teukolsky, S. A., Vetterling, W. T., \& Flannery, B. P. (2007). Numerical Recipes: The Art of Scientific Computing. New York: Cambridge University Press. [Richardson et al. 2003] Richardson, M. J. E., Brunel, N., \& Hakim, V. (2003). From subthreshold to firing-rate resonance. Journal of Neurophysiology, 89, 2538-2554.

[Samengo et al. 2013] Samengo, I., Elijah, D., \& Montemurro, M. A. (2013). Spike-train analysis. In R. Quian Quiroga \& S. Panzeri (Eds.), Principles of Neural Coding. Boca Raton: CRC Press.

[Samengo and Gollisch 2013] Samengo, I., \& Gollisch, T. (2013). Spike-triggered covariance: Geometric proof, symmetry properties, and extension beyond Gaussian stimuli. Journal Computational Neuroscience, 34, 137-161.

[Sanchez-Vives et al. 2000] Sanchez-Vives, M. V., Nowak, L. G., \& McCormick, D. A. (2000). Cellular mechanisms of long-lasting adaptation in visual cortical neurons in vitro. Journal of 
Neuroscience, 20(11), 4286-4299.

[Schwartz et al. 2007] Schwartz, O., Hsu, A., \& Dayan, P. (2007). Space and time in visual context. Nature Reviews Neuroscience, 8, 522-535.

[Schwartz and Simoncelli 2001] Schwartz, O., \& Simoncelli, E. P. (2001). Natural signal statistics and sensory gain control. Nature Neuroscience, 4(8), 819-825.

[Segev et al. 2006] Segev, R., Puchalla, J., \& Berry II, M. J. (2006). Functional organization of ganglion cells in the salamander retina. Journal of Neurophysiology, 95, 2277-2292.

[Sharpee et al. 2008] Sharpee, T. O., Miller, K. D., \& Stryker, M. P. (2008). On the importance of static nonlinearity in estimating spatiotemporal neural filters with natural stimuli. Journal of Neurophysiology, 99, 2496-2509.

[Sharpee et al. 2011] Sharpee, T. O., Nagel, K. I., \& Doupe, A. J. (2011). Two-dimensional adaptation in the auditory forebrain. Journal of Neurophysiology, 106, 1841-1861.

[Shu et al. 2003] Shu, Y., Hasenstaub, A., \& McCormick, D. A. (2003). Turning on and off recurrent balanced cortical activity. Nature, 423, 288-293.

[Sjöström et al. 2008] Sjöström, P. J., Rancz, E. A., Roth, A., \& Häusser, M. (2008). Dendritic excitability and synaptic plasticity. Physiological Reviews, 88, 769-840.

[Theunissen et al. 2001] Theunissen, F. E., David, S. V., Singh, N. C., Hsu, A., Vinje, W. E., \& Gallant, J. L. (2001). Estimating spatio-temporal receptive fields of auditory and visual neurons from their responses to natural stimuli. Network: Computation in Neural Systems, 12, 289-316.

[Theunissen et al. 2000] Theunissen, F. E., Sen, K., \& Doupe, A. J. (2000). Spectral-temporal receptive fields of nonlinear auditory neurons obtained using natural sounds. Journal of Neuroscience, 20(6), 2315-2331.

[Ulanovsky et al. 2003] Ulanovsky, N., Las, L., \& Nelken, I. (2003). Processing of low-probability sounds by cortical neurons. Nature Neuroscience, 6(4), 391-398.

[Urdapilleta 2011] Urdapilleta, E. (2011). Onset of negative interspike interval correlations in adapting neurons. Physical Review E, 84, 041904.

[Urdapilleta and Samengo 2009] Urdapilleta, E., \& Samengo, I. (2009). The firing statistics of Poisson neuron models driven by slow stimuli. Biological Cybernetics, 101, 265-277.

[Victor 1987] Victor, J. D. (1987). The dynamics of the cat retinal X cell centre. Journal of Physiology, 386, 219-246. 
[Wang 1998] Wang, X. J. (1998). Calcium coding and adaptive temporal computation in cortical pyramidal neurons. Journal of Neurophysiology, 79, 1549-1566.

[Wang 2010] Wang, X. J. (2010). Neurophysiological and computational principles of cortical rhythms in cognition. Physiological Reviews, 90, 1195-1268.

[Wark et al. 2009] Wark, B., Fairhall, A., \& Rieke, F. (2009). Timescales of inference in visual adaptation. Neuron, 61, 750-761.

[Wark et al. 2007] Wark, B., Lundstrom, B. N., \& Fairhall, A. (2007). Sensory adaptation. Current Opinion in Neurobiology, 17, 423-429.

[Wilson and Cowan 1972] Wilson, R., \& Cowan, J. D. (1972). Excitatory and inhibitory interactions in localized populations of model neurons. Biophysical Journal, 12, 1-24.

[Xu-Friedman and Regehr 2004] Xu-Friedman, M. A., \& Regehr, W. G. (2004). Structural contributions to short-term synaptic plasticity. Physiological Reviews, 84, 69-85. 